\title{
TORIC BRUHAT INTERVAL POLYTOPES
}

\author{
EUNJEONG LEE, MIKIYA MASUDA, AND SEONJEONG PARK
}

\begin{abstract}
For two elements $v$ and $w$ of the symmetric group $\mathfrak{S}_{n}$ with $v \leq w$ in Bruhat order, the Bruhat interval polytope $Q_{v, w}$ is the convex hull of the points $(z(1), \ldots, z(n)) \in \mathbb{R}^{n}$ with $v \leq z \leq w$. It is known that the Bruhat interval polytope $Q_{v, w}$ is the moment map image of the Richardson variety $X_{w^{-1}}^{v^{-1}}$. We say that $Q_{v, w}$ is toric if the corresponding Richardson variety $X_{w^{-1}}^{v^{-1}}$ is a toric variety. We show that when $Q v, w$ is toric, its combinatorial type is determined by the poset structure of the Bruhat interval $[v, w]$ while this is not true unless $Q_{v, w}$ is toric. We are concerned with the problem of when $Q_{v, w}$ is (combinatorially equivalent to) a cube because $Q_{v, w}$ is a cube if and only if $X_{w^{-1}}^{v^{-1}}$ is a smooth toric variety. We show that a Bruhat interval polytope $Q_{v, w}$ is a cube if and only if $Q_{v, w}$ is toric and the Bruhat interval [v,w] is a Boolean algebra. We also give several sufficient conditions on $v$ and $w$ for $Q_{v, w}$ to be a cube.
\end{abstract}

\section{CONTENTS}

1. Introduction

2. Preliminaries

3. Relation with Richardson varieties

4. Properties of Bruhat interval polytopes

5. Toric Bruhat interval polytopes

6. Product of Bruhat intervals

7. Conditions on $v$ and $w$ for $Q_{v, w}$ to be toric

8. Finding all coatoms in some special cases

References

\section{INTRODUCTION}

The permutohedron Perm ${ }_{n-1}$ is an $(n-1)$-dimensional simple polytope in $\mathbb{R}^{n}$ defined by the convex hull of all points $(u(1), u(2), \ldots, u(n)) \in \mathbb{R}^{n}$ for $u$ in the symmetric group $\mathfrak{S}_{n}$ on the set $\{1,2, \ldots, n\}$. It was first investigated by Schoute in 1911 (see 24 and references therein), and later Guilbaud and Rosenstiehl gave the name "permutohedron" in [10. There are many works on generalizations of the notion of permutohedra such as generalized permutohedra in [17, graphicahedra in 2, Bruhat interval polytopes in 22, and so on.

On the other hand, the permutohedra have appeared in not only combinatorics but also the geometries of flag varieties. The flag variety $\mathcal{F} \ell_{n}$ is a smooth projective variety which consists of chains $\{0\} \subset V_{1} \subset V_{2} \subset \cdots \subset V_{n}=\mathbb{C}^{n}$ of subspaces of $\mathbb{C}^{n}$ with $\operatorname{dim}_{\mathbb{C}} V_{i}=i$. It is known that the algebraic torus $\mathbb{T}=\left(\mathbb{C}^{*}\right)^{n}$ acts on $\mathcal{F} \ell_{n}$ and there is a one-to-one correspondence between the set of

Date: May 7, 2019.

2010 Mathematics Subject Classification. Primary: 14M25, 14M15, secondary: 05A05.

Key words and phrases. Bruhat interval polytopes, Richardson varieties, toric varieties. 
fixed points of $\mathbb{T}$ on $\mathcal{F} \ell_{n}$ and the elements of $\mathfrak{S}_{n}$. Moreover, the moment map image of $\mathcal{F} \ell_{n}$ is the permutohedron Perm $_{n-1}$, and the closure of the $\mathbb{T}$-orbit of a generic point of $\mathcal{F} \ell_{n}$ is known to be the permutohedral variety, which is the toric variety whose fan is the normal fan of Perm ${ }_{n-1}$ (see [13, 18,).

In this manuscript, we are studying Bruhat interval polytopes that were introduced by Tsukerman and Williams 22 in 2015. For two elements $v$ and $w$ of the symmetric group $\mathfrak{S}_{n}$ with $v \leq w$ in Bruhat order, the Bruhat interval polytope $Q_{v, w}$ is defined to be the convex hull of all points $(z(1), \ldots, z(n)) \in \mathbb{R}^{n}$ with $v \leq z \leq w$. Bruhat interval polytopes are one of the generalizations of permutohedra. Indeed, the Bruhat interval polytope $Q_{e, w_{0}}$ is the permutohedron Perm $_{n-1}$ where $e$ is the identity element and $w_{0}$ is the longest element in $\mathfrak{S}_{n}$.

As in the case of permutohedra and flag varieties, Bruhat interval polytopes are related with Richardson varieties. For $v \leq w$, the Richardson variety $X_{w}^{v}$ is defined to be the intersection of the Schubert variety $X_{w}$ and the opposite Schubert variety $w_{0} X_{w_{0} v}$. It is an irreducible $\mathbb{T}$-invariant subvariety of the flag variety $\mathcal{F} \ell_{n}$. It is known that there is a one-to-one correspondence between the set of fixed points of $\mathbb{T}$ on the Richardson variety $X_{w}^{v}$ and the set $\{z \mid v \leq z \leq w\}$, and it leads naturally to consider the convex hull of the points $(z(1), \ldots, z(n)) \in \mathbb{R}^{n}$ with $v \leq z \leq w$. Note that the moment map image of the Richardson variety $X_{w}^{v}$ is the Bruhat interval polytope $Q_{v^{-1}, w^{-1}}$ not $Q_{v, w}$ (see Lemma 3.1).

It should be noted that Bruhat interval polytopes $Q_{v, w}$ and $Q_{v^{-1}, w^{-1}}$ are not combinatorially equivalent in general even though the Bruhat intervals $[v, w]$ and $\left[v^{-1}, w^{-1}\right]$ are isomorphic as posets. Moreover, even if two Bruhat interval polytopes $Q_{v, w}$ and $Q_{v^{-1}, w^{-1}}$ are combinatorially equivalent, the fact that a subinterval $[x, y] \subset[v, w]$ is realized as a face of $Q_{v, w}$ does not imply that the subinterval $\left[x^{-1}, y^{-1}\right]$ is realized as a face of $Q_{v^{-1}, w^{-1}}$ (see Remark 4.4).

We are particularly interested in Bruhat interval polytopes whose corresponding Richardson varieties are toric varieties (note that $X_{w}^{v}$ is a toric variety if and only if so is $X_{w^{-1}}^{v^{-1}}$ ). We call such a Bruhat interval polytope toric. It is known that $\operatorname{dim} Q_{v, w} \leq \operatorname{dim} X_{w^{-1}}^{v^{-1}}=\ell(w)-\ell(v)$ in general, where $\ell\left(\right.$ ) denotes the length of a permutation, and we have that $\operatorname{dim} Q_{v, w}=\ell(w)-\ell(v)$ if and only if $Q_{v, w}$ is toric (see Section 3). Toric Bruhat interval polytopes have a bunch of nice properties that an arbitrary Bruhat interval polytope does not have. Furthermore, those nice properties give us topological and geometric information of toric Richardson varieties.

Theorem 1.1 (Theorem 5.1). A Bruhat interval polytope $Q_{v, w}$ is toric if and only if every subinterval $[x, y]$ of $[v, w]$ is realized as a face of $Q_{v, w}$.

The above theorem implies that if $Q_{v, w}$ is toric, then its combinatorial type is determined by the poset structure of $[v, w]$, and hence $Q_{v, w}$ and $Q_{v^{-1}, w^{-1}}$ are combinatorially equivalent.

Combinatorial properties of a toric Bruhat interval polytope $Q_{v, w}$ give us some geometric information about the toric Richardson variety $X_{w}^{v}$. The toric Richardson variety $X_{w}^{v}$ is smooth at a $\mathbb{T}$-fixed point $u B$ for $v \leq u \leq w$ if and only if the vertex $u$ of the Bruhat interval polytope $Q_{v, w}$ is simple, that is, the number of edges meeting at the vertex $u$ is same as the dimension of the polytope $Q_{v, w}$ (see Proposition 4.77). Hence a Richardson variety is a smooth toric variety if and only if the corresponding Bruhat interval polytope is toric and a simple polytope.

Note that every toric Schubert variety is smooth and its corresponding Bruhat interval polytope is combinatorially equivalent to a cube (see [7, 12, 15]). But not every toric Bruhat interval polytope is a simple polytope and hence not every toric Richardson variety is smooth. See Figures 1 and 3 , By restricting our attention to toric Bruhat interval polytopes, we get the following.

Proposition 1.2 (Proposition [5.6). A toric Bruhat interval polytope is a simple polytope if and only if it is combinatorially equivalent to a cube. 
It is well-known in toric topology that every smooth toric variety whose fan is the normal fan of a combinatorial cube has a sequence of $\mathbb{C} P^{1}$-fiber bundles, so called a Bott tower 11 Hence the above proposition implies that every smooth toric Richardson variety is a Bott manifold that is a manifold in a Bott tower. We can further show the following whose geometric meaning is that a Richardson variety $X_{w}^{v}$ is a Bott manifold if and only if it is toric and the Bruhat interval $[v, w]$ is a Boolean algebra.

Theorem 1.3 (Theorem [5.7). A Bruhat interval polytope $Q_{v, w}$ is combinatorially equivalent to a cube if and only if it is toric and the Bruhat interval $[v, w]$ is a Boolean algebra.

In the above theorem, we cannot drop the toric condition. There exist permutations $v$ and $w$ in $\mathfrak{S}_{n}(n \geq 4)$ such that the Bruhat interval $[v, w]$ is a Boolean algebra but the combinatorial type of the Bruhat interval polytope $Q_{v, w}$ is not a cube. See Figure 8 and Section 6 .

We also study a necessary and sufficient condition on $v$ and $w$ such that the Bruhat interval polytope $Q_{v, w}$ is toric or combinatorially equivalent to a cube. It was shown in [7 that a Bruhat interval polytope $Q_{e, w}$ is combinatorially equivalent to a cube if and only if $w$ is a product of distinct simple transpositions. But the similar extension does not hold for general $v$. That is, even if there exist reduced expressions $r(v)$ and $r(w)$ for $v$ and $w$ such that the subword $r(w) \backslash r(v)$ of $r(w)$ consists of distinct simple transpositions, we cannot conclude that $Q_{v, w}$ is combinatorially equivalent to a cube (see Example 5.9) nor toric (see Example 7.10). So, it seems difficult to characterize $v$ and $w$ for which $Q_{v, w}$ is toric or combinatorially equivalent to a cube. We find some sufficient conditions on $v$ and $w$ for $Q_{v, w}$ to be toric, and give a necessary and sufficient condition on $v$ and $w$ for $Q_{v, w}$ to be a cube when $v$ and $w$ satisfy some special condition.

This manuscript is organized as follows. In Section 2, we compile some basic facts on posets, polytopes and toric varieties, and introduce Bruhat interval polytopes. In Section 3 we show that the Bruhat interval polytope $Q_{v, w}$ is the moment map image of the Richardson variety $X_{w^{-1}}^{v^{-1}}$. In Section 4 we interpret combinatorial properties of Bruhat interval polytopes in terms of graphs defined by Bruhat intervals. Section 5 deals with properties of toric Bruhat interval polytopes and contains the proof of Theorem 1.3. In Section 6, we show that there are infinitely many non-simple toric Bruhat interval polytopes. In Section 7 , we find some sufficient conditions on $v$ and $w$ for $Q_{v, w}$ to be toric, and then for such toric Bruhat interval polytopes $Q_{v, w}$ we find a sufficient condition to be a cube. In Section 8 , we will find all coatoms of the Bruhat interval $[v, w]$ when $v$ and $w$ satisfy some special condition, and then describe when $Q_{v, w}$ is a cube for such special cases.

Acknowledgements. The authors thanks to Akiyoshi Tsuchiya for his computer program to check Conjecture 4.8 for $\mathfrak{S}_{5}$ and $\mathfrak{S}_{6}$. Lee was supported by IBS-R003-D1. Masuda was supported in part by JSPS Grant-in-Aid for Scientific Research 16K05152. Park was supported by Basic Science Research Program through the National Research Foundation of Korea (NRF) funded by the Government of Korea (NRF-2018R1A6A3A11047606) and (NRF-2016R1D1A1A09917654).

\section{Preliminaries}

In this section, we prepare some notions and basic facts about posets and polytopes, and then introduce the notion of Bruhat interval polytopes.

\footnotetext{
${ }^{1} \mathrm{~A}$ Bott tower is a family of smooth projective toric varieties $\left\{B_{2 k} \mid 1 \leq k \leq n\right\}$ such that $B_{2}=\mathbb{C} P^{1}$ and $B_{2 k}=$ $P\left(\underline{\mathbb{C}} \oplus \xi_{k-1}\right)$ for $1<k \leq n$ where $P(\cdot)$ denotes complex projectivization, $\xi_{k-1}$ is a complex line bundle over $B_{2(k-1)}$ and $\underline{\mathbb{C}}$ is the trivial line bundle (see $[9]$ ). We call $B_{2 k}$ a Bott manifold (of height $k$ ).
} 
2.1. Posets and Bruhat orders. Let $\mathcal{P}$ be a poset (partially ordered set) with an order relation $<$. For two elements $x, y \in \mathcal{P}$, we say $y$ covers $x$, denoted by $x \lessdot y$, if $x<y$ and there is no $z$ such that $x<z<y$. We also call it a cover $x \lessdot y$. One represents $\mathcal{P}$ as a mathematical diagram, called a Hasse diagram, in a way that a point in the plane is drawn for each element of $\mathcal{P}$, and a line segment or curve is drawn upward from $x$ to $y$ whenever $y$ covers $x$. A chain of $\mathcal{P}$ is a totally ordered subset $\sigma$ of $\mathcal{P}$, and the length $\ell(\sigma)$ of a chain $\sigma$ is defined to be $|\sigma|-1$. The length $\ell(\mathcal{P})$ of a poset $\mathcal{P}$ is the length of a longest chain of $\mathcal{P}$. For $x \leq y$ in $\mathcal{P}$, let $[x, y]$ denote the closed interval $\{z \in \mathcal{P} \mid x \leq z \leq y\}$, and let $(x, y)$ denote the open interval $\{z \in \mathcal{P} \mid x<z<y\}$. If $\mathcal{P}$ has a unique minimum element, it is referred to as the bottom element. Similarly, the unique maximum element, if it exists, is referred to as the top element. An element of $\mathcal{P}$ that covers the bottom element is called an atom; and an element covered by the top element is called a coatom.

A graded poset is a poset $\mathcal{P}$ equipped with a rank function $\rho$ from $\mathcal{P}$ to $\mathbb{Z}_{\geq 0}$ satisfying the following:

(1) if $x<y$ in $\mathcal{P}$, then $\rho(x)<\rho(y)$; and

(2) if $x \lessdot y$, then $\rho(y)=\rho(x)+1$.

The value of the rank function for an element of the poset is called its rank.

Let $\mathfrak{S}_{n}$ be the symmetric group on the set $[n]:=\{1,2, \ldots, n\}$. We will denote an element $v \in \mathfrak{S}_{n}$ by

$$
[v(1), v(2), \ldots, v(n)] \quad \text { or } \quad v(1) v(2) \cdots v(n) .
$$

For $1 \leq i<j \leq n$, the permutation which acts on $[n]$ by swapping $i$ and $j$ is called a transposition and denoted by $(i, j)$ or $t_{i, j}$. Indeed,

$$
(i, j)=t_{i, j}=[1,2, \ldots, i-1, j, i+1, \ldots, j-1, i, j+1, \ldots, n] .
$$

We denote the set of transpositions in $\mathfrak{S}_{n}$ by $T$.

$$
T=\{(i, j) \mid 1 \leq i<j \leq n\} .
$$

The simple transpositions $s_{i}$ are the transpositions of the form

$$
s_{i}:=(i, i+1), \quad \text { for } i=1, \ldots, n-1 .
$$

Note that every element of $\mathfrak{S}_{n}$ can be represented as a product of simple transpositions, although the decomposition is not unique.

For $v \in \mathfrak{S}_{n}$, if $v=s_{i_{1}} \cdots s_{i_{\ell}}$ and is minimal among all such expressions, then the string of indices $i_{1} \cdots i_{\ell}$ is called a reduced decomposition of $v$ and $\ell$ is called the length of $v$, denoted $\ell(v)$. Note that $\ell\left(v^{-1}\right)=\ell(v)$. The Bruhat order on $\mathfrak{S}_{n}$ is defined by $v \leq w$ if a reduced decomposition for $v$ is a substring of some reduced decomposition for $w$. Then the Bruhat order on $\mathfrak{S}_{n}$ is a graded poset, with rank function given by length. The elements

$$
e:=[1,2, \ldots, n] \quad \text { and } \quad w_{0}:=[n, n-1, \ldots, 1]
$$

are the bottom and the top elements of the poset $\mathfrak{S}_{n}$, respectively. For $v$ and $w$ in $\mathfrak{S}_{n}$ with $v \leq w$, the Bruhat interval $[v, w]$ is defined to be the closed interval

$$
[v, w]=\left\{z \in \mathfrak{S}_{n} \mid v \leq z \leq w\right\} .
$$

Figure $1(\mathrm{a})$ shows the Hasse diagram of $\mathfrak{S}_{4}$ under Bruhat order and we illustrate an example of a Bruhat interval in Figure 1(b) 


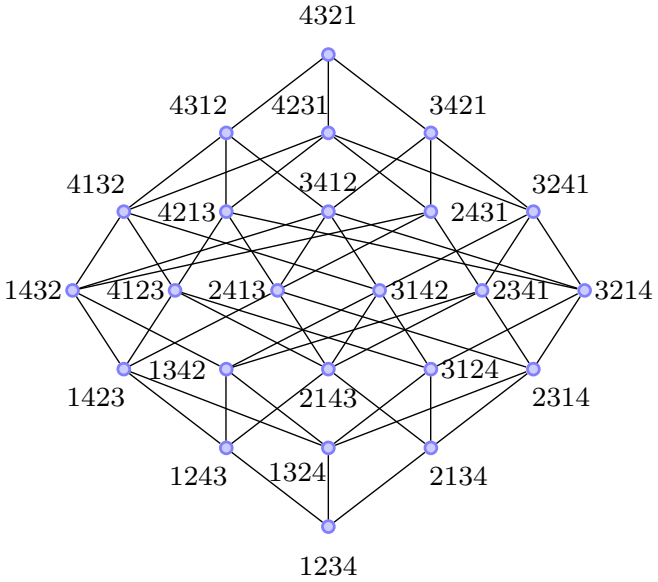

(a) Bruhat order of $\mathfrak{S}_{4}$.

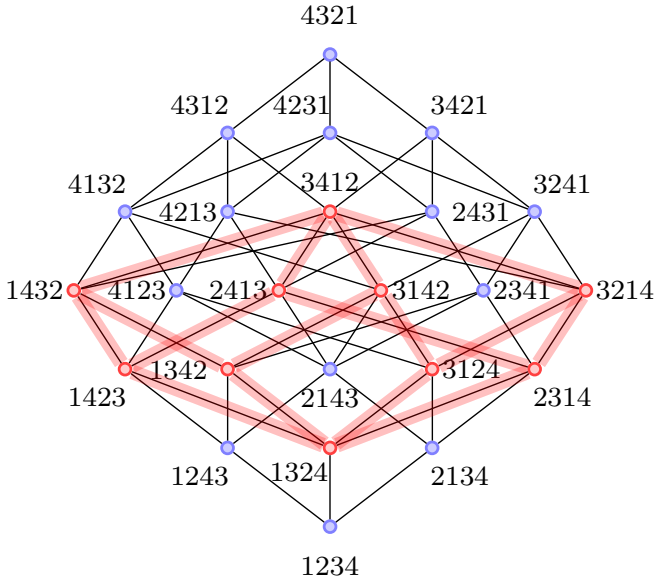

(b) Bruhat interval [1324, 3412].

Figure 1. Bruhat order of $\mathfrak{S}_{4}$ and an example of a Bruhat interval.

2.2. Polytopes and toric varieties. A convex polytope is the convex hull of a finite set of points in the Euclidean space $\mathbb{R}^{n}$. It is well known that every convex polytope is a bounded intersection of finitely many half-spaces. Two polytopes are combinatorially equivalent if their face posets are isomorphic. For a vertex $v$ of a polytope $P$, the degree $d(v)$ of $v$ is the number of edges meeting at $v$. For an $n$-dimensional polytope $P$, a vertex $v$ of $P$ is said to be simple if $d(v)=n$. When all the vertices of $P$ are simple, we call $P$ a simple polytope.

A lattice polytope is a convex polytope whose vertices are in the lattice $\mathbb{Z}^{n} \subset \mathbb{R}^{n}$. A vertex $v$ of a lattice polytope $P$ is said to be smooth if it is simple and the primitive direction vectors of the edges emanating from $v$ form a basis for $\mathbb{Z}^{n}$. We call a vertex of $P$ singular if it is not smooth. A lattice polytope $P$ is said to be smooth if all the vertices of $P$ are smooth. We call a lattice polytope $P$ is singular if some vertex of $P$ is singular. See Figure 2 ,

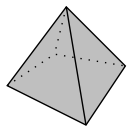

(a) Not simple (so singular).

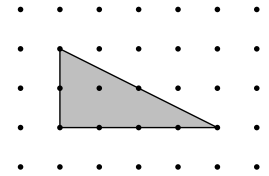

(b) Simple but singular.

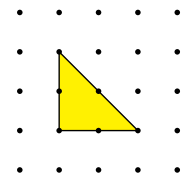

(c) Smooth.

FIgURE 2. Example and non-examples of smooth lattice polytopes.

A toric variety of complex dimension $n$ is a normal algebraic variety containing an algebraic torus $\left(\mathbb{C}^{*}\right)^{n}$ as a Zariski open dense subset such that the action of the torus on itself extends to the whole variety. It is known that a lattice polytope $P$ defines a projective toric variety $X(P)$, that is, $X(P)$ can be given as the closure of the image of a map $\left(\mathbb{C}^{*}\right)^{n} \rightarrow \mathbb{C} P^{\ell}$, defined by Laurent monomials as in [6, Proposition 3.1.6]. Moreover, the vertices of $P$ correspond to the $\mathbb{T}$-fixed points of $X(P)$, and a vertex $v$ of $P$ is smooth if and only if $X(P)$ is smooth at the corresponding fixed point. 
It was shown in [16. Corollary 3.5] that if a smooth lattice polytope $P$ is combinatorially equivalent to a cube, then the toric variety $X(P)$ is weakly equivariantly diffeomorphic to a Bott manifold (see the footnote in the introduction for Bott manifolds).

2.3. Bruhat interval polytope. The notion of Bruhat interval polytopes was introduced by Tsukerman and Williams [22] as a natural generalization of permutohedra.

Definition 2.1. For elements $v$ and $w$ in $\mathfrak{S}_{n}$ with $v \leq w$, the Bruhat interval polytope $Q_{v, w}$ is the convex hull of all permutation vectors $z=(z(1), z(2), \ldots, z(n)) \in \mathbb{R}^{n}$ with $v \leq z \leq w$.

By definition, every Bruhat interval polytope is a lattice polytope and hence it defines a projective toric variety.

Note that the Bruhat interval polytope $Q_{e, w_{0}}$ is the permutohedron Perm ${ }_{n-1}$, the convex hull of the $n$ ! points obtained by permuting the coordinates of the vector $(1,2, \ldots, n)$. Two vertices $(v(1), \ldots, v(n))$ and $(w(1), \ldots, w(n))$ are joined by an edge in the permutohedron if and only if there exists a simple transposition $s_{i}$ such that $w=s_{i} v$ (see Figure 3(a)). Furthermore, the permutohedron Perm ${ }_{n-1}$ defines a smooth projective toric variety called the permutohedral variety. But not every toric variety defined by a Bruhat interval polytope is smooth. For example, the polytope $Q_{1324,3412}$ is not a simple polytope (see Figure 3(b)].

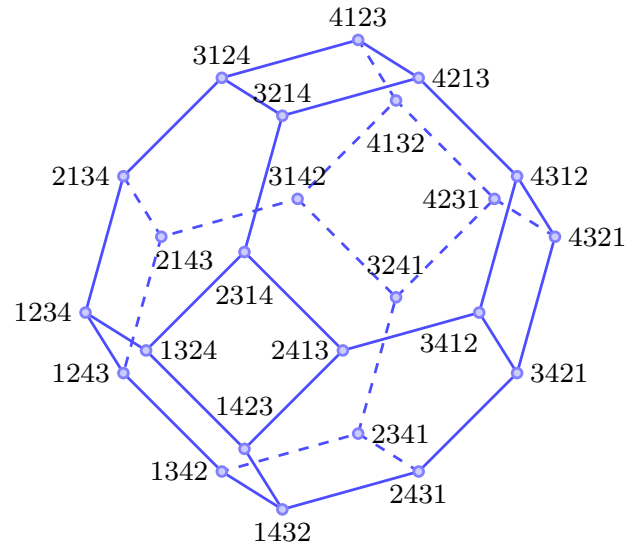

(a) $\operatorname{Perm}_{3}\left(w\right.$ and $s_{i} w$ are joined by an edge).

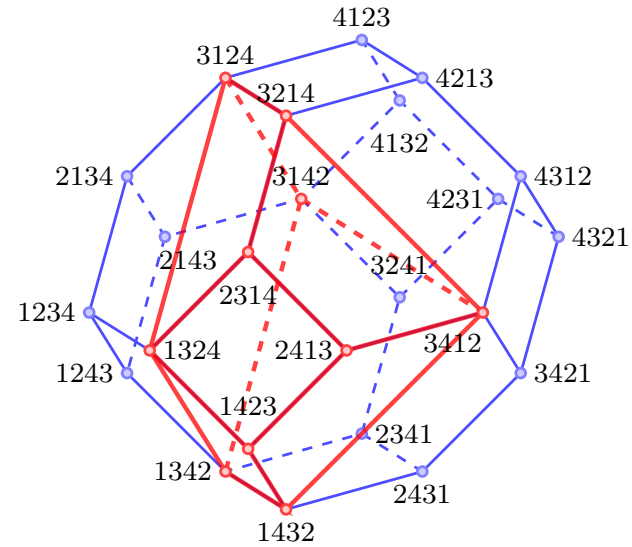

(b) Bruhat interval polytope $Q_{1324,3412}$.

Figure 3. Permutohedron Perm 3 and an example of a Bruhat interval polytope.

\section{Relation With Richardson varieties}

In this section, we review the relation between Bruhat interval polytopes and Richardson varieties, and introduce the connection between combinatorial properties of Bruhat interval polytopes and geometric properties of Richardson varieties.

Let $G=\mathrm{GL}_{n}(\mathbb{C}), B \subset G$ the set of upper triangular matrices, and $\mathbb{T} \subset G$ the set of diagonal matrices. Let $B^{-} \subset G$ be the set of lower triangular matrices. Then $\mathbb{T}:=B \cap B^{-}$and $B^{-}=w_{0} B w_{0}$. The manifold $G / B$ can be identified with the flag variety $\mathcal{F} \ell_{n}$ which is defined to be

$$
\mathcal{F} \ell_{n}:=\left\{\left(\{0\} \subset V_{1} \subset V_{2} \subset \cdots \subset V_{n}=\mathbb{C}^{n}\right) \mid \operatorname{dim}_{\mathbb{C}} V_{i}=i \quad \text { for all } i=1, \ldots, n\right\} .
$$

For an element $w \in \mathfrak{S}_{n}$, we define the permutation matrix $\left[\begin{array}{lll}\mathbf{e}_{w(1)} & \cdots & \mathbf{e}_{w(n)}\end{array}\right] \in \mathrm{GL}_{n}(\mathbb{C})$ where $\mathbf{e}_{1}, \ldots, \mathbf{e}_{n}$ are the standard basis vectors in $\mathbb{R}^{n}$. We will write it simply $w$ if there is no confusion. 
For an element $w \in \mathfrak{S}_{n}$, we denote the Schubert variety $\overline{B w B / B}$ (respectively, the opposite Schubert variety $\overline{B^{-} w B / B}$ ) in the flag variety $G / B$ by $X_{w}$ (respectively, $X^{w}$ ). The left multiplication by $\mathbb{T}$ on $G$ induces the $\mathbb{T}$-action on $G / B$ which leaves both $X_{w}$ and $X^{w}$ invariant. The set of $\mathbb{T}$-fixed points in $G / B$ bijectively corresponds to the symmetric group $\mathfrak{S}_{n}$ through the correspondence $u \in \mathfrak{S}_{n} \rightarrow u B \in G / B$. A fixed point $u B$ is contained in $X_{w}$ if and only if $u \leq w$ in Bruhat order and $u B$ is contained in $X^{w}$ if and only if $u \geq w$ in Bruhat order (see [8, $\left.\S 10.5\right]$ ).

For elements $v$ and $w \in \mathfrak{S}_{n}$ with $v \leq w$, we define the Richardson variety $X_{w}^{v}$ by $X^{v} \cap X_{w}$. Then $X_{w_{0}}^{e}=G / B, X_{w}^{e}=X_{w}$, and $X_{w_{0}}^{w}=X^{w}$. Furthermore, $X_{w}^{v}$ is also $\mathbb{T}$-invariant and the $\mathbb{T}$-fixed points of $X_{w}^{v}$ correspond to the elements in the Bruhat interval $[v, w]$. It is known that

$$
\operatorname{dim}_{\mathbb{C}} X_{w}^{v}=\ell(w)-\ell(v),
$$

see [5].

The full flag variety $G / B$ has the symplectic form $\omega_{\lambda}$ due to Kirillov, Kostant, and Souriau for a regular dominant weight $\lambda$. When we choose the weight $\lambda$ as the sum of all fundamental weights, the permutohedron Perm $_{n-1}$ is the moment map image of $G / B$ (see, for example, [3, Corollary IV.4.11] and references therein). Now we describe the moment map $\mu: G / B \rightarrow \mathbb{R}^{n}$ explicitly using the Plücker coordinates. We define the set

$$
I_{d, n}=\left\{\underline{\mathbf{i}}=\left(i_{1}, \ldots, i_{d}\right) \in \mathbb{Z}^{d} \mid 1 \leq i_{1}<\cdots<i_{d} \leq n\right\} .
$$

For an element $x=\left(x_{i j}\right) \in G=\mathrm{GL}_{n}(\mathbb{C})$, the $\underline{\mathbf{i}}$ th Plücker coordinate $p_{\mathbf{i}}(x)$ of $x$ is given by the $d \times d$ minor of $x$, with row indices $i_{1}, \ldots, i_{d}$ and the column indices $1, \ldots, d$ for $\underline{\mathbf{i}}=\left(i_{1}, \ldots, i_{d}\right) \in I_{d, n}$. Then the Plücker embedding is defined to be

$$
\psi: G / B \rightarrow \prod_{d=1}^{n-1} \mathbb{C} P^{\left(\begin{array}{l}
n \\
d
\end{array}\right)-1}, \quad x B \mapsto \prod_{d=1}^{n-1}\left(p_{\underline{\mathbf{i}}}(x)\right)_{\underline{\mathbf{i}} \in I_{d, n}} .
$$

The map $\psi$ is $\mathbb{T}$-equivariant with respect to the action of $\mathbb{T}$ on $\prod_{d=1}^{n-1} \mathbb{C} P^{\left(\begin{array}{l}n \\ d\end{array}\right)-1}$ given by

$$
\left(t_{1}, \ldots, t_{n}\right) \cdot\left(p_{\underline{\mathbf{i}}}\right)_{\mathbf{i} \in I_{d, n}}:=\left(t_{i_{1}} \cdots t_{i_{d}} \cdot p_{\underline{\mathbf{i}}}\right)_{\mathbf{i} \in I_{d, n}}
$$

for $\left(t_{1}, \ldots, t_{n}\right) \in \mathbb{T}$ and $\underline{\mathbf{i}}=\left(i_{1}, \ldots, i_{d}\right)$. Then the moment map $\tilde{\mu}: \prod_{d=1}^{n-1} \mathbb{C} P^{\left(\begin{array}{l}n \\ d\end{array}\right)-1} \rightarrow \mathbb{R}^{n}$ is given by

$$
\left(p_{\underline{\mathbf{i}}}\right)_{\underline{\mathbf{i}} \in I_{d, n}} \mapsto-\sum_{d=1}^{n-1}\left\{\frac{1}{\sum_{\underline{\mathbf{i}} \in I_{d, n}}\left|p_{\underline{\mathbf{i}}}\right|^{2}}\left(\sum_{1 \in \mathbf{i} \in I_{d, n}}\left|p_{\mathbf{i}}\right|^{2}, \ldots, \sum_{n \in \underline{\mathbf{i}} \in I_{d, n}}\left|p_{\underline{\mathbf{i}}}\right|^{2}\right)\right\}+\mathbf{c}
$$

where $\mathbf{c}$ is a constant vector. By setting $\mathbf{c}=(n, \ldots, n)$ in (3.3) and $\mu:=\tilde{\mu} \circ \psi$, we can see the following.

Lemma 3.1. The moment map $\mu$ sends the fixed point $u B \in G / B$ to $\left(u^{-1}(1), \ldots, u^{-1}(n)\right) \in \mathbb{R}^{n}$.

Proof. For a permutation $u \in \mathfrak{S}_{n}$, the Plücker coordinates $\left(p_{\underline{\mathbf{i}}}\right)_{\underline{\mathbf{i}} \in I_{d, n}}$ of $u B$ are given as follows:

$$
p_{\underline{\mathbf{i}}}= \begin{cases}1 & \text { if } \underline{\mathbf{i}}=\{u(1), \ldots, u(d)\} \uparrow \\ 0 & \text { otherwise }\end{cases}
$$

for each $\underline{\mathbf{i}} \in I_{d, n}$. Here, for a subset $S \subset[n]$, we denote by $S \uparrow$ the ordered tuple obtained from $S$ by sorting its elements in ascending order. Therefore one can see that for a fixed $d \in[n-1]$, $\sum_{\underline{\mathbf{i}} \in I_{d, n}}\left|p_{\mathbf{i}}\right|^{2}=1$ and the vector

$$
\left(\sum_{1 \in \mathbf{i} \in I_{d, n}}\left|p_{\mathbf{i}}\right|^{2}, \ldots, \sum_{n \in \mathbf{i} \in I_{d, n}}\left|p_{\mathbf{i}}\right|^{2}\right)
$$


becomes an integer vector whose entries are 1 for coordinates in $\{u(1), \ldots, u(d)\}$ and 0 otherwise. Hence the summation

$$
-\sum_{d=1}^{n-1}\left\{\frac{1}{\sum_{\underline{\mathbf{i}} \in I_{d, n}}\left|p_{\mathbf{i}}\right|^{2}}\left(\sum_{1 \in \underline{\mathbf{i}} \in I_{d, n}}\left|p_{\mathbf{i}}\right|^{2}, \ldots, \sum_{n \in \underline{\mathbf{i}} \in I_{d, n}}\left|p_{\mathbf{i}}\right|^{2}\right)\right\}
$$

is an integer vector such that the $u(k)$-entry is $-(n-k)$. Therefore, the moment map image $\mu(u B)$ is an integer vector whose $u(k)$-entry is $k$ since $\mathbf{c}=(n, \cdots, n)$ in (3.2). This implies that $\mu(u B)=\left(u^{-1}(1), \ldots, u^{-1}(n)\right)$ since $u^{-1}(u(k))=k$ for all $k$.

It follows from Lemma 3.1 that for $v$ and $w \in \mathfrak{S}_{n}$ with $v \leq w$, the Bruhat interval polytope $Q_{v, w}$ is the moment map image of the Richardson variety $X_{w^{-1}}^{v^{-1}}$.

Example 3.2. Suppose that $G=\mathrm{GL}_{3}(\mathbb{C})$. Then the Plücker embedding $\psi: G / B \rightarrow \mathbb{C} P^{\left(\begin{array}{l}3 \\ 1\end{array}\right)-1} \times$ $\mathbb{C} P^{\left(\begin{array}{l}3 \\ 2\end{array}\right)-1}$ maps an element $x=\left(x_{i j}\right) \in \mathrm{GL}_{3}(\mathbb{C})$ to

$$
\begin{aligned}
& \left(\left[p_{1}(x), p_{2}(x), p_{3}(x)\right],\left[p_{1,2}(x), p_{1,3}(x), p_{2,3}(x)\right]\right) \\
& \quad=\left(\left[x_{11}, x_{21}, x_{31}\right],\left[x_{11} x_{22}-x_{21} x_{12}, x_{11} x_{32}-x_{31} x_{12}, x_{21} x_{32}-x_{31} x_{22}\right]\right) .
\end{aligned}
$$

Since the action of $\mathbb{T}$ on $\mathrm{GL}_{3}(\mathbb{C})$ is given by

$$
\left(t_{1}, t_{2}, t_{3}\right) \cdot\left(\begin{array}{lll}
x_{11} & x_{12} & x_{13} \\
x_{21} & x_{22} & x_{23} \\
x_{31} & x_{32} & x_{33}
\end{array}\right)=\left(\begin{array}{lll}
t_{1} x_{11} & t_{1} x_{12} & t_{1} x_{13} \\
t_{2} x_{21} & t_{2} x_{22} & t_{2} x_{23} \\
t_{3} x_{31} & t_{3} x_{32} & t_{3} x_{33}
\end{array}\right),
$$

one can easily check that the map $\psi$ is $\mathbb{T}$-equivariant. The moment map $\tilde{\mu}: \mathbb{C} P^{\left(\begin{array}{l}3 \\ 1\end{array}\right)-1} \times \mathbb{C} P^{\left(\begin{array}{l}3 \\ 2\end{array}\right)-1} \rightarrow \mathbb{R}^{3}$ is given by

$$
\begin{aligned}
\left(\left[p_{1}, p_{2}, p_{3}\right],\left[p_{12}, p_{13}, p_{23}\right]\right) & \\
\mapsto & -\frac{1}{\left|p_{1}\right|^{2}+\left|p_{2}\right|^{2}+\left|p_{3}\right|^{2}}\left(\left|p_{1}\right|^{2},\left|p_{2}\right|^{2},\left|p_{3}\right|^{2}\right) \\
& -\frac{1}{\left|p_{12}\right|^{2}+\left|p_{13}\right|^{2}+\left|p_{23}\right|^{2}}\left(\left|p_{12}\right|^{2}+\left|p_{13}\right|^{2},\left|p_{12}\right|^{2}+\left|p_{23}\right|^{2},\left|p_{13}\right|^{2}+\left|p_{23}\right|^{2}\right) \\
& +(3,3,3) .
\end{aligned}
$$

Then one can see that

$$
\mu(312 B)=\tilde{\mu} \circ \psi(312 B)=\tilde{\mu}(([0,0,1],[0,1,0]))=-(0,0,1)-(1,0,1)+(3,3,3)=(2,3,1) .
$$

We call a $\mathbb{T}$-orbit in $X_{w}^{v}$ generic if its closure contains all the $\mathbb{T}$-fixed points in $X_{w}^{v}$ and call a point in $X_{w}^{v}$ generic if it is in a generic $\mathbb{T}$-orbit. We will denote the closure of a generic $\mathbb{T}$-orbit in $X_{w}^{v}$ by $Y_{w}^{v}$. Then $Y_{w}^{v}$ is the projective toric variety defined by the polytope $Q_{v^{-1}, w^{-1}}$. Hence it follows from (3.1) that

$$
\operatorname{dim} Q_{v, w}=\operatorname{dim}_{\mathbb{C}} Y_{w^{-1}}^{v^{-1}} \leq \operatorname{dim}_{\mathbb{C}} X_{w^{-1}}^{v^{-1}}=\ell\left(w^{-1}\right)-\ell\left(v^{-1}\right)=\ell(w)-\ell(v) .
$$

Motivated by this observation, we introduce the following terminology.

Definition 3.3. The Bruhat interval polytope $Q_{v, w}$ is called toric if $\operatorname{dim} Q_{v, w}=\ell(w)-\ell(v)$.

Equation (3.4) implies that the Richardson variety $X_{w}^{v}$ is a toric variety, that is, $X_{w}^{v}=Y_{w}^{v}$, if and only if the Bruhat interval polytope $Q_{v^{-1}, w^{-1}}$ is toric. In general, Bruhat interval polytopes $Q_{v, w}$ and $Q_{v^{-1}, w^{-1}}$ are not combinatorially equivalent even though $x \lessdot y$ is a cover in $[v, w]$ if and only if $x^{-1} \lessdot y^{-1}$ is a cover in $\left[v^{-1}, w^{-1}\right]$ (see Remark 4.4). But they have the same dimension. We get the following whose proof will be given in the next section. 
Proposition 3.4. Two Bruhat interval polytopes $Q_{v, w}$ and $Q_{v^{-1}, w^{-1}}$ have the same dimension. In particular, $X_{w}^{v}$ is a toric variety if and only if so is $X_{w^{-1}}^{v^{-1}}$. Indeed, the Richardson variety $X_{w}^{v}$ is a toric variety if and only if $Q_{v, w}$ is toric.

\section{Properties of Bruhat interval polytopes}

In this section, we review some notations and facts about Bruhat interval polytopes and related graphs introduced in [15] and 22. Then we interpret combinatorial properties of Bruhat interval polytopes using these graphs. Using this interpretation, we provide a proof of Proposition 3.4 which shows that Bruhat interval polytopes $Q_{v, w}$ and $Q_{v^{-1}, w^{-1}}$ have the same dimension.

We first set up notations and terminologies related to digraphs (or directed graphs). A digraph is an ordered pair $G=(V(G), E(G))$, where

- $V(G)$ is a set whose elements are called vertices, and

- $E(G)$ is a multiset of ordered pairs of vertices, called directed edges.

For two vertices $i$ and $j$ of a given graph $G, i$ can reach $j$ if there is a (directed) path from $i$ to $j$. A digraph $G$ is said to be acyclic if there is no directed cycle. The underlying graph of $G$ is the undirected graph created using all of the vertices in $V(G)$ and replacing all directed edges in $E(G)$ with undirected edges. A digraph is connected (or weakly connected) if the underlying graph is a connected graph. Hence if $i$ can reach $j$, then $i$ and $j$ are connected, but the converse is not true in general. If $V(G)=[n]$, then we can define $B(G)$ to be a partition of the set $[n]$ such that each block corresponds to a connected component of $G$.

Let $v, w \in \mathfrak{S}_{n}$ with $v \leq w$. For $u \in[v, w]$, we define the following two sets:

$$
\begin{aligned}
& \bar{T}(u,[v, w])=\{t \in T \mid \exists z \stackrel{t}{\rightarrow} u, z \in[v, w]\}=\{t \in T \mid u \lessdot u t \leq w\} \text { and } \\
& \underline{T}(u,[v, w])=\{t \in T \mid \exists z \stackrel{t}{\leftarrow} u, z \in[v, w]\}=\{t \in T \mid v \leq u t \lessdot u\} .
\end{aligned}
$$

Here, $T$ is the set of transpositions (see (2.1)). The digraph $G_{u}^{v, w}$ is defined as follows:

(1) The vertices of $G_{u}^{v, w}$ are $\{1,2, \ldots, n\}$.

(2) There is a directed edge from $i$ to $j$ for every $(i, j) \in \bar{T}(u,[v, w])$.

(3) There is a directed edge from $j$ to $i$ for every $(i, j) \in \underline{T}(u,[v, w])$.

Then the dimension of the Bruhat interval polytope $Q_{v, w}$ is determined by the number of blocks of the partition determined by the graph $G_{v}^{v, w}$ or $G_{w}^{v, w}$, that is,

$$
\operatorname{dim} Q_{v, w}=n-\# B\left(G_{v}^{v, w}\right)=n-\# B\left(G_{w}^{v, w}\right),
$$

see Theorem 4.6 and Proposition 4.10 of $[22$.

Example 4.1. Let $[v, w]=[1324,4231]$. Then the digraph $G_{v}^{v, w}$ is connected (see Figure 4) and the Bruhat interval polytope $Q_{v, w}$ is of dimension 3.

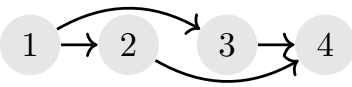

FiguRE 4. Graph $G_{1324}^{1324,4231}$.

Now we give the proof of Proposition 3.4 which claims that two Bruhat interval polytope $Q_{v, w}$ and $Q_{v^{-1}, w^{-1}}$ have the same dimension. 
Proof of Proposition 3.4. Let $\bar{T}(v,[v, w])=\left\{\left(i_{1}, j_{1}\right),\left(i_{2}, j_{2}\right), \ldots,\left(i_{k}, j_{k}\right)\right\}$. Since $\underline{T}(v,[v, w])=\emptyset$, we get $E\left(G_{v}^{v, w}\right)=\bar{T}(v,[v, w])$. Since $(i, j) v^{-1}=v^{-1}(v(i), v(j))$ for every transposition $(i, j) \in T$, we have that

$$
E\left(G_{v^{-1}}^{v^{-1}, w^{-1}}\right)=\bar{T}\left(v^{-1},\left[v^{-1}, w^{-1}\right]\right)=\left\{\left(v\left(i_{1}\right), v\left(j_{1}\right)\right),\left(v\left(i_{2}\right), v\left(j_{2}\right)\right), \ldots,\left(v\left(i_{k}\right), v\left(j_{k}\right)\right)\right\} .
$$

Since $v$ is a bijection on $[n]$, the graph $G_{v}^{v, w}$ is isomorphic to $G_{v^{-1}}^{v^{-1}, w^{-1}}$, and hence the partitions $B\left(G_{v}^{v, w}\right)$ and $B\left(G_{v^{-1}}^{v^{-1}}, w^{-1}\right)$ consist of the same number of blocks. Therefore, $\operatorname{dim} Q_{v, w}=$ $\operatorname{dim} Q_{v^{-1}, w^{-1}}$ by (4.1).

Even though $Q_{v, w}$ and $Q_{v^{-1}, w^{-1}}$ can have the same dimension, their face structures cannot be compared with each other in general (see Remark 4.4). Now, we define a digraph $G_{x, y}^{v, w}$ for each $[x, y] \subset[v, w]$ and use it to verify that $Q_{x, y}$ is a face of $Q_{v, w}$. The digraph $G_{x, y}^{v, w}$ is defined as follows:

(1) The vertices of $G_{x, y}^{v, w}$ are $\{1,2, \ldots, n\}$, with nodes $i$ and $j$ identified if they are in the same block of $B\left(G_{x}^{x, y}\right)$.

(2) There is a directed edge from $i$ to $j$ for every $(i, j) \in \bar{T}(y,[v, w])$.

(3) There is a directed edge from $j$ to $i$ for every $(i, j) \in \underline{T}(x,[v, w])$.

It has been known from [22, Theorem 4.1] that every face of a Bruhat interval polytope is itself a Bruhat interval polytope. Moreover, for $[x, y] \subset[v, w]$, one can determine whether the Bruhat interval polytope $Q_{x, y}$ is a face of $Q_{v, w}$ by checking the acyclicity of $G_{x, y}^{v, w}$.

Theorem 4.2 ([22, Theorem 4.19]). For $[x, y] \subset[v, w]$, the Bruhat interval polytope $Q_{x, y}$ is a face of the Bruhat interval polytope $Q_{v, w}$ if and only if the graph $G_{x, y}^{v, w}$ is an acyclic digraph.

Example 4.3. Let $[v, w]=[1324,4231]$ and consider $[x, y]=[1432,2431] \subset[v, w]$. Note that $\left[v^{-1}, w^{-1}\right]=[v, w]$ and $\left[x^{-1}, y^{-1}\right]=[1432,4132]$. Since $y=x(1,4), B\left(G_{x}^{x, y}\right)=14|2| 3$ and $B\left(G_{x^{-1}}^{x^{-1}, y^{-1}}\right)=12|3| 4$. We have that

$$
\bar{T}(y,[v, w])=\{(1,2)\} \quad \text { and } \quad \underline{T}(x,[v, w])=\{(2,3),(3,4)\},
$$

and the graph $G_{x, y}^{v, w}$ is acyclic (see Figure $5(\mathrm{a})$. On the other hand, we get

$$
\bar{T}\left(y^{-1},\left[v^{-1}, w^{-1}\right]\right)=\{(2,4)\} \quad \text { and } \quad \underline{T}\left(x^{-1},\left[v^{-1}, w^{-1}\right]\right)=\{(2,3),(3,4)\},
$$

so that the graph $G_{x^{-1}, y^{-1}}^{v^{-1}, w^{-1}}$ is a directed cycle (see Figure $5(\mathrm{~b})$.

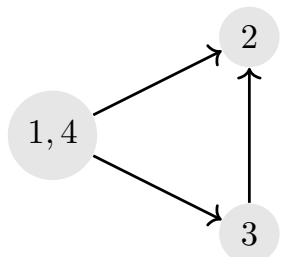

(a) $G_{1432,2431}^{1324,4231}$ : acyclic.

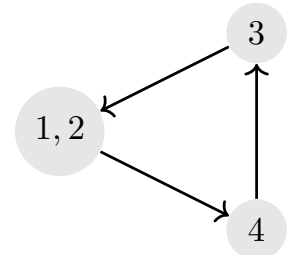

(b) $G_{1432,4132}^{1324,4231}$ : directed cycle.

FIGURE 5. $G_{x, y}^{v, w}$ is acyclic but $G_{x^{-1}, y^{-1}}^{v^{-1}, w^{-1}}$ is not.

Remark 4.4. The graph $G_{1432,2431}^{1324,4231}$ is acyclic but the graph $G_{1432,4132}^{1324,4231}$ is not as in Example 4.3. Therefore, the fact that $Q_{x, y}$ is a face of $Q_{v, w}$ does not imply that $Q_{x^{-1}, y^{-1}}$ is a face of $Q_{v^{-1}, w^{-1}}$. See Figure 3(b) for the Bruhat interval polytope $Q_{1324,3412}$. Moreover, the Bruhat interval polytopes $Q_{v, w}$ and $Q_{v^{-1}, w^{-1}}$ are not combinatorially equivalent in general. For example, one can check that 
two Bruhat interval polytopes $Q_{12345,35412}$ and $Q_{12345,45132}$ are not combinatorially equivalent using a computer program, for example, using SAGE.

A transitive reduction of a digraph $G$ is another digraph with the same vertices and as few edges as possible, such that if there is a directed path from vertex $i$ to vertex $j$, then there is also such a path in the reduction. That is, the reduction has the same reachability relations as $G$. Remarkably, the transitive reduction of a finite acyclic digraph is unique and is a subgraph of the given graph. We can find the transitive reduction of a finite acyclic digraph by removing each directed edge $i \rightarrow j$ if there is a directed path from $i$ to $j$. See [1] for more details.

Example 4.5. A transitive reduction of the graph in Figure 4 is itself. Now consider the graphs in Example 4.3. For the graph in Figure 5(a) the node $\{1,4\}$ reaches to the node $\{2\}$ via two different ways: $\{1,4\} \rightarrow\{2\}$ and $\{1,4\} \rightarrow\{3\} \rightarrow\{2\}$. Since this graph is a finite acyclic digraph, it has a unique transitive reduction (see Figure 6(a). On the other hand, the graph $G_{1432,4132}^{1324,4231}$ in Figure 5(b) and the graph given in Figure 6(b) have the same reachability relations, and those graphs are a transitive reduction of each other

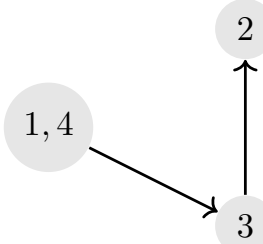

(a) The transitive reduction of Figure $5(\mathrm{a})$

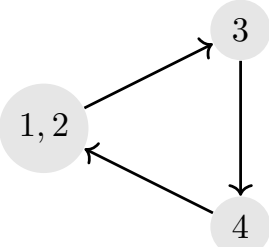

(b) A transitive reduction of Figure 5(b)

FiguRE 6. Transitive reductions.

Since every $u \in[v, w]$ can be realized as a vertex of the polytope $Q_{v, w}$, the graph $G_{u}^{v, w}$ is an acyclic digraph by Theorem 4.2. Hence $G_{u}^{v, w}$ has a unique transitive reduction and we denote it by $\widetilde{G}_{u}^{v, w} 3$ Note that a directed edge from $i$ to $j$ in $G_{u}^{v, w}$ is disappeared in $\widetilde{G}_{u}^{v, w}$ if and only if the vector $\mathbf{e}_{i}-\mathbf{e}_{j}$ can be expressed as a sum of vectors in the set $\left\{\mathbf{e}_{k}-\mathbf{e}_{\ell} \mid(k, \ell) \in E\left(\widetilde{G}_{u}^{v, w}\right)\right\}$. Hence $Q_{v, w}$ is contained in

$$
(u(1), \ldots, u(n))+\operatorname{Cone}\left(\left\{\mathbf{e}_{i}-\mathbf{e}_{j} \mid(i, j) \in E\left(\widetilde{G}_{u}^{v, w}\right)\right\}\right) .
$$

Corollary 4.6 (see [22, Corollary 4.20]). Let $v, w \in \mathfrak{S}_{n}$ with $v \leq w$. For $u \in[v, w]$, the number of edges meeting at the vertex $u$ in $Q_{v, w}$ equals the number of edges in $\widetilde{G}_{u}^{v, w}$. Furthermore, the primitive direction vectors of the edge emanating from a vertex $u$ of $Q_{v, w}$ are given by $\mathbf{e}_{i}-\mathbf{e}_{j}$ for a directed edge from $i$ to $j$ in the graph $\widetilde{G}_{u}^{v, w}$.

We can check the smoothness of a vertex of a Bruhat interval polytope by using the graph $\widetilde{G}_{u}^{v, w}$.

Proposition 4.7. Let $v$ and $w$ be in $\mathfrak{S}_{n}$ with $v \leq w$ and let $u \in[v, w]$. The following are equivalent:

(1) The vertex $u$ is simple in $Q_{v, w}$.

(2) The vertex $u$ is smooth in $Q_{v, w}$.

(3) The undirected underlying graph of $\widetilde{G}_{u}^{v, w}$ is a forest.

${ }^{2}$ For this reason, uniqueness of a transitive reduction fails for digraphs with cycles.

${ }^{3}$ Note that the graph $\widetilde{G}_{u^{-1}}^{e, w^{-1}}$ is equal to the graph $\Gamma_{w}(u)$ in $[15$ for $u \leq w$. 
Proof. The proof of [15, Lemma 6.6] works here too.

The following proposed conjecture is a generalized version of Conjecture 7.16 in [15].

Conjecture 4.8. The polytope $Q_{v, w}$ is a simple polytope if and only if the vertices $v$ and $w$ are simple. Equivalently, if two graphs $\widetilde{G}_{v}^{v, w}$ and $\widetilde{G}_{v}^{v, w}$ are forests, then $\widetilde{G}_{u}^{v, w}$ is a forest for every $v \leq u \leq w$.

In fact, we have computer-based evidence [21 that the conjecture above is true for $n \leq 6$.

Note that the dimension of the cone in (4.2) is independent of the choice of $u$ since it is equal to the dimension of the polytope $Q_{v, w}$. This says that $\# B\left(\widetilde{G}_{u}^{v, w}\right)$ is independent of the choice of $u$.

Corollary 4.9. Let $v, w \in \mathfrak{S}_{n}$ and $v \leq w$ and $u \in[v, w]$. The number of the connected components of the graph $\widetilde{G}_{u}^{v, w}$ is independent of $u$, and $\operatorname{dim} Q_{v, w}=n-\# B\left(G_{u}^{v, w}\right)$.

Proof. Recall that the incidence matrix of a connected digraph on $[n]$ has rank $(n-1)$. Let $M_{u}$ be the incidence matrix of the graph $\widetilde{G}_{u}^{v, w}$. Then the rank of $M_{u}$ is equal to the dimension of the cone in (4.2), and hence it is equal to the dimension of $Q_{v, w}$. Therefore,

$$
\operatorname{dim} Q_{v, w}=\operatorname{rank}\left(M_{u}\right)=n-\# B\left(\widetilde{G}_{u}^{v, w}\right) .
$$

Since $B\left(\widetilde{G}_{u}^{v, w}\right)=B\left(G_{u}^{v, w}\right)$, this proves the corollary.

We can further prove that the partition $B\left(\widetilde{G}_{u}^{v, w}\right)$ is independent of $u$ (see Proposition 4.12). To give a proof of this, we introduce an operation on partitions.

For partitions $P$ and $Q$ of $[n]$, we define a partition $P * Q$ of $[n]$ as follows: two elements $i, j \in[n]$ are in a same block of $P * Q$ if and only if there is a sequence $i=i_{1}, i_{2}, \ldots, i_{k}=j$ such that each consecutive pair $\left(i_{\ell}, i_{\ell+1}\right)(\ell=1,2, \ldots, k-1)$ is in a same block of either $P$ or $Q$.

Example 4.10. Let

$$
\begin{aligned}
& P=\{\{1\},\{2,3,4\},\{5\},\{6,7\},\{8\},\{9,10\}\} \\
& Q=\{\{1,3\},\{2,4\},\{5,7\},\{6,8\},\{9,10\}\}
\end{aligned}
$$

be partitions on [10]. Then

$$
P * Q=\{\{1,2,3,4\},\{5,6,7,8\},\{9,10\}\} .
$$

Note that if $G$ and $H$ are (undirected) graphs on $[n]$, then $B(G) * B(H)$ coincides with the partition determined by the graph sum of $G$ and $H$, the graph with adjacency matrix given by the sum of adjacency matrices of $G$ and $H$.

We introduce another way to compute the dimension of a Bruhat interval polytope by using a graph $G^{\mathcal{E}}$. Let $v, w \in \mathfrak{S}_{n}$ with $v \leq w$, and let $\mathcal{C}: v=x_{(0)} \lessdot x_{(1)} \lessdot \cdots \lessdot x_{(\ell)}=w$ be a maximal chain from $v$ to $w$. Then recall from [22, §4.2] that the undirected graph $G^{\mathcal{C}}$ on $[n]$ is defined to be the graph whose edge set is the set of unordered pairs

$$
\left\{\{a, b\} \mid(a, b)=x_{(i)}^{-1} x_{(i+1)} \in T \quad \text { for some } 0 \leq i \leq \ell-1\right\} .
$$

Here, $T$ is the set of transpositions (see (2.1)). Note that $G^{\mathcal{C}}$ can have multiple edges. It was shown in [22, Corollary 4.8] that $B\left(G^{\mathcal{C}}\right)$ is independent of the choice of $\mathcal{C}$, and denoted by $B_{v, w}$. Furthermore, the dimension of the polytope $Q_{v, w}$ is determined by the partition $B_{v, w}$,

$$
\operatorname{dim} Q_{v, w}=n-\# B_{v, w}
$$

see [22, Theorem 4.6]. It was also shown in [22, Corollary 4.10] that

$$
B\left(G_{v}^{v, w}\right)=B\left(G_{w}^{v, w}\right)=B_{v, w} .
$$


Example 4.11. Suppose that $v=1324=s_{2}$ and $w=4231=s_{3} s_{2} s_{1} s_{2} s_{3}$. Choose a maximal chain e from $v$ to $w$ :

$$
\begin{array}{cccccccccc}
\mathcal{C}: & s_{2} & \lessdot & s_{3} s_{2} & \lessdot & s_{3} s_{2} s_{1} & \lessdot & s_{3} s_{2} s_{1} s_{2} & \lessdot & s_{3} s_{2} s_{1} s_{2} s_{3} . \\
\| & & \| & & \| & & \| & & \| \\
& x_{(0)} & & x_{(1)} & & x_{(2)} & & x_{(3)} & & x_{(4)}
\end{array}
$$

Then we have that

$$
x_{(0)}^{-1} x_{(1)}=s_{2} s_{3} s_{2}=(2,4), \quad x_{(1)}^{-1} x_{(2)}=s_{1}=(1,2), \quad x_{(2)}^{-1} x_{(3)}=s_{2}=(2,3), \quad x_{(3)}^{-1} x_{(4)}=s_{3}=(3,4) .
$$

Hence the corresponding graph is given as in Figure 7 and the partition $B_{v, w}$ is $\{[4]\}$. Therefore, the Bruhat interval polytope $Q_{1324,4231}$ is of dimension 3.

$$
1-2 \simeq 3 \simeq 4
$$

Figure 7. Graph $G^{\mathcal{C}}$ for the chain $\mathcal{C}$ in (4.5).

Proposition 4.12. Let $v, w \in \mathfrak{S}_{n}$ and $v \leq w$ and $u \in[v, w]$. The connected components of the graph $\widetilde{G}_{u}^{v, w}$ are independent of $u$. Furthermore, $B\left(\widetilde{G}_{u}^{v, w}\right)=B_{v, w}$.

Proof. Note that $B\left(G_{u}^{v, w}\right)=B\left(\widetilde{G}_{u}^{v, w}\right)$. Then we can see that

$$
\begin{aligned}
B\left(\widetilde{G}_{u}^{v, w}\right) & =B\left(\widetilde{G}_{u}^{v, u}\right) * B\left(\widetilde{G}_{u}^{u, w}\right) \\
& \left.=B_{v, u} * B_{u, w} \quad \text { (by (4.4) }\right) .
\end{aligned}
$$

Let us choose a maximal chain $\mathcal{C}$ of $[v, w]$ containing $u$. That is,

$$
\mathcal{C}: v=x_{(0)} \lessdot x_{(1)} \lessdot \cdots \lessdot x_{(k)} \lessdot \cdots \lessdot x_{(\ell)}=w \text { and } x_{(k)}=u \text {. }
$$

If $\mathcal{C}_{-}: x_{(0)} \lessdot x_{(1)} \lessdot \cdots \lessdot x_{(k)}$ and $\mathcal{C}_{+}: \lessdot x_{(k)} \lessdot \cdots \lessdot x_{(\ell)}$ are the subchains of $\mathcal{C}$, then $B_{v, u}=B\left(G^{\mathcal{C}_{-}}\right)$ and $B_{u, w}=B\left(G^{\mathcal{C}_{+}}\right)$. Since $B\left(G^{\mathcal{C}}\right)=B\left(G^{\mathcal{C}_{-}}\right) * B\left(G^{\mathcal{C}_{+}}\right)$, we conclude that $B_{v, u} * B_{u, w}=B_{v, w}$.

\section{Toric Bruhat interval polytopes}

Recall that a Bruhat interval polytope $Q_{v, w}$ is toric if $\operatorname{dim} Q_{v, w}=\ell(w)-\ell(v)$. In this section, we show that the combinatorial type of a toric Bruhat interval polytope $Q_{v, w}$ is determined by the poset structure of the interval $[v, w]$ (see Theorem 5.1). Furthermore, a toric Bruhat interval polytope is simple if and only if it is combinatorially equivalent to a cube (see Corollary 5.12).

We already have seen in Theorem 4.2 that every face of a Bruhat interval polytope $Q_{v, w}$ can be realized by a subinterval of $[v, w]$. We can show that the converse is also true when $Q_{v, w}$ is toric.

Theorem 5.1. For a Bruhat interval polytope $Q_{v, w}$, the following are equivalent:

(1) $Q_{v, w}$ is toric (i.e., $\operatorname{dim} Q_{v, w}=\ell(w)-\ell(v)$ ).

(2) $Q_{x, y}$ is a face of $Q_{v, w}$ for any $[x, y] \subset[v, w]$.

Proof. Suppose that (1) holds. Since $Q_{v, w}$ is toric, the Richardson variety $X_{w^{-1}}^{v^{-1}}$ is a toric variety by definition. We note that $\left[x^{-1}, y^{-1}\right] \subset\left[v^{-1}, w^{-1}\right]$ if (and only if) $[x, y] \subset[v, w]$. Since $X_{y^{-1}}^{x^{-1}}$ is a toric subvariety of $X_{w^{-1}}^{v^{-1}}$ and the moment map gives a one-to-one correspondence between toric subvarieties of $X_{w^{-1}}^{v^{-1}}$ and faces of $Q_{v, w}$, we have that $\mu\left(X_{y^{-1}}^{x^{-1}}\right)=Q_{x, y}$ is a face of $\mu\left(X_{w^{-1}}^{v^{-1}}\right)=Q_{v, w}$, proving (2).

Conversely, suppose (2) holds. We shall prove (1) by induction on the value of $\ell(w)-\ell(v)$. When $\ell(w)-\ell(v)=1,(1)$ obviously holds. We note that for any $[p, q] \subset[x, y], Q_{p, q}$ is a face of $Q_{x, y}$ by $(2)$. 


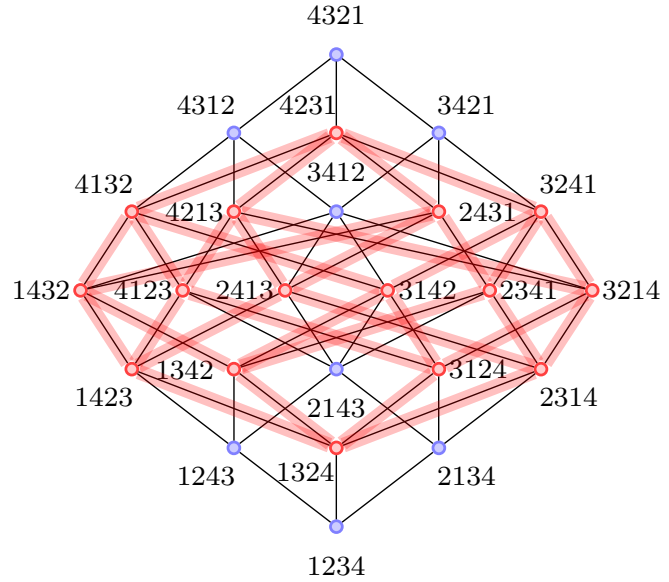

(a) Bruhat interval [1324, 4231].

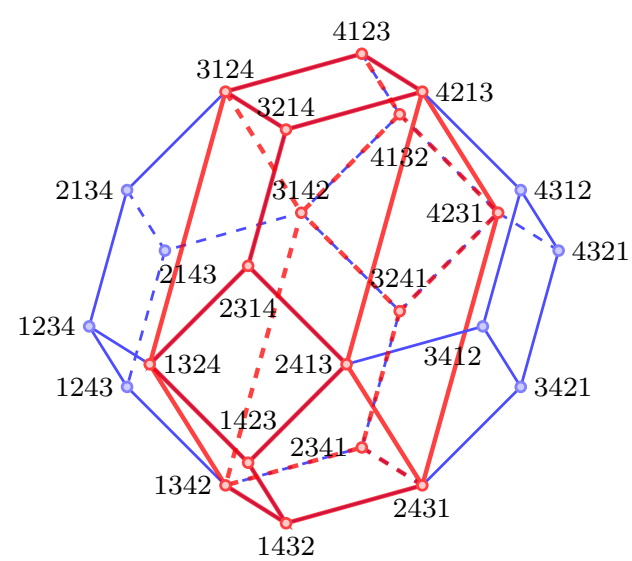

(b) Bruhat interval polytope $Q_{1324,4231}$.

Figure 8 . The interval $[1324,4231]$ is a Boolean algebra but $Q_{1324,4231}$ is not a cube.

If $[x, y]$ is a proper subset of $[v, w]$, then $\ell(y)-\ell(x)<\ell(w)-\ell(v)$ and hence $\operatorname{dim} Q_{x, y}=\ell(y)-\ell(x)$ by induction assumption.

Now we take $x=v$ and $y \lessdot w$. Then

$$
\operatorname{dim} Q_{v, y}=\ell(w)-\ell(y)=\ell(w)-\ell(v)-1 .
$$

Moreover, since $Q_{v, y}$ is a face of $Q_{v, w}$ by (2) and does not contain the vertex $w$, we have

$$
\operatorname{dim} Q_{v, w}>\operatorname{dim} Q_{v, y} .
$$

It follows from (5.1) and (5.2) that $\operatorname{dim} Q_{v, w} \geq \ell(w)-\ell(v)$. Since the converse inequality holds by (3.4), this proves (1).

Theorem [5.1 shows that if $Q_{v, w}$ is toric, then the poset structure of $[v, w]$ determines the face poset of $Q_{v, w}$. Hence the following corollary directly follows.

Corollary 5.2. If $Q_{v, w}$ is toric, then its combinatorial type is determined by the poset structure of $[v, w]$.

The assumption "toric" in the corollary above cannot be removed. Indeed, the interval [1324, 4231] is a Boolean algebra of rank 4 but the corresponding Bruhat interval polytope is of dimension 3. See Figure 8. On the other hand, for each positive integer $m$ there is an example of an interval $[v, w]$ of rank $m$ such that $[v, w]$ is a Boolean algebra and $Q_{v, w}$ is toric (so that $\operatorname{rank}[v, w]=\operatorname{dim} Q_{v, w}$ ), e.g. $w=s_{m} s_{m-1} \cdots s_{2} s_{1}$ and $v=e$. In fact, $Q_{v, w}$ of this example is combinatorially equivalent to an $m$-cube.

Corollary 5.3. If $Q_{v, w}$ is toric, then $Q_{v, w}$ and $Q_{v^{-1}, w^{-1}}$ are combinatorially equivalent.

Remark 5.4. For $n \leq 4$ and for every pair of $v$ and $w$ in $\mathfrak{S}_{n}$ with $v \leq w, Q_{v, w}$ is combinatorially equivalent to $Q_{v^{-1}, w^{-1}}$. But for $n=5$ there are 160 pairs of $v$ and $w$ such that $Q_{v, w}$ is not combinatorially equivalent to $Q_{v^{-1}, w^{-1}}$. 


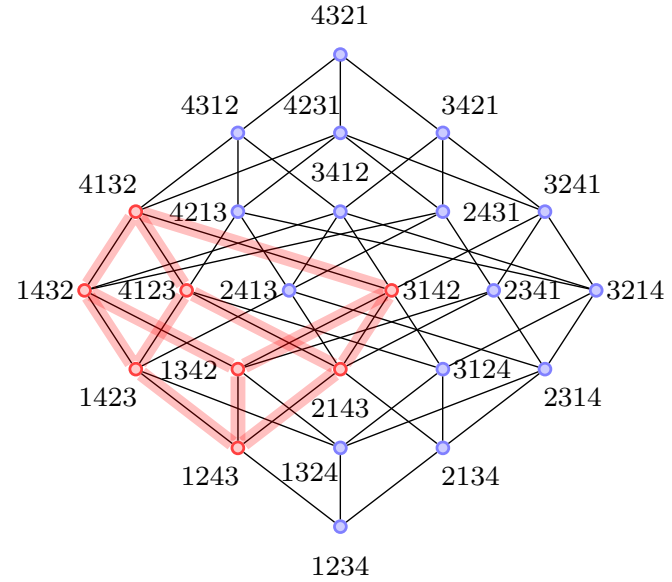

(a) Bruhat interval [1243, 4132].

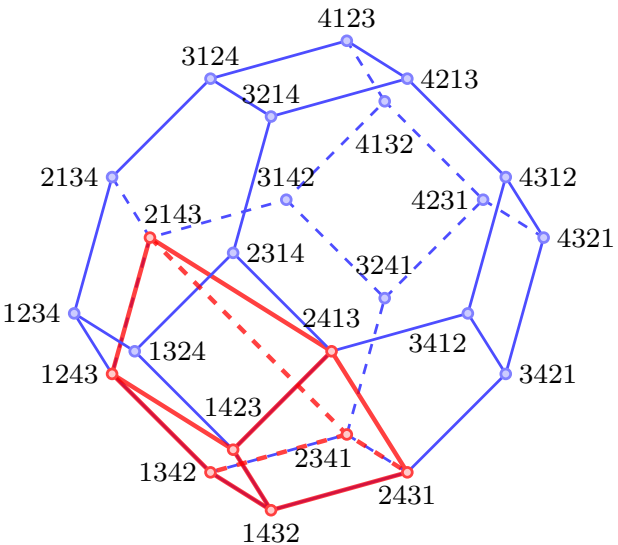

(b) Bruhat interval polytope $Q_{1243,2431}$.

Figure 9. An example of a Bruhat interval polytope which is a cube.

Convention. In the following, when a polytope $Q$ is combinatorially equivalent to a cube (or a $d$-cube), we simply say that $Q$ is a cube (or a $d$-cube). We also say that an interval $[v, w]$ is Boolean if it is a Boolean algebra.

We recall the following fact from [24, Problems and Exercises 0.1 in p.23] (see also [23, Appendix]):

Lemma 5.5. If $Q$ is a simple polytope of dimension $\geq 2$ and every 2 -face of $Q$ is a 2-cube, then $Q$ is a cube.

This lemma implies the following.

Proposition 5.6. Suppose that $Q_{v, w}$ is toric. Then $Q_{v, w}$ is a cube if and only if it is simple. (This is equivalent to saying that a smooth toric Richardson variety is a Bott manifold.)

Proof. Since the "only if" part is trivial, it suffices to prove the "if "part. Since $Q_{v, w}$ is toric, every $k$-face of $Q_{v, w}$ corresponds to a $k$-interval in $[v, w]$. Since every 2-interval is a diamond (see [4, Lemma 2.7.3]), every 2-face of $Q_{v, w}$ is a 2-cube. Therefore, if $Q_{v, w}$ is simple, then $Q_{v, w}$ is a cube by Lemma 5.5

The following gives a characterization of when $Q_{v, w}$ is a cube and also proves Theorem 1.3

Theorem 5.7. A Bruhat interval polytope $Q_{v, w}$ is a cube if and only if it is toric and $[v, w]$ is Boolean. (This is equivalent to saying that a Richardson variety $X_{w}^{v}$ is a Bott manifold if and only if it is toric and $[v, w]$ is Boolean.)

In Figure 9, one can see an example of a Bruhat interval polytope $Q_{v, w}$ which is toric and the interval $[v, w]$ is Boolean. Combining the above theorem with Corollary [5.3, we get the following.

Corollary 5.8. A Bruhat interval polytope $Q_{v, w}$ is a cube if and only if $Q_{v^{-1}, w^{-1}}$ is a cube.

Proof of Theorem [5.7. First we prove the "if" part. Since $[v, w]$ is Boolean, every element $u \in[v, w]$ covers $\ell(u)-\ell(v)$ elements and is covered by $\ell(w)-\ell(u)$ elements. Since $Q_{v, w}$ is toric, $\operatorname{dim} Q_{v, w}=$ $\ell(w)-\ell(v)$ and any edge incident to the vertex $u$ in $Q_{v, w}$ is obtained from the cover relations by Theorem [5.1. This shows that $Q_{v, w}$ is simple. Therefore, $Q_{v, w}$ is a cube by Proposition 5.6. 
The rest of the proof is devoted to the proof of the "only if" part. We shall prove it by induction on the dimension $m$ of the cube. It is obvious when $m=1$. Suppose that it holds for $m-1$ and that $Q_{v, w}$ is an $m$-cube. Then $Q_{v, w}$ has two disjoint facets, both of which are an $(m-1)$-cube. We denote those facets by $Q_{p, q}$ and $Q_{r, s}$. Then

(1) $[p, q] \cap[r, s]=\emptyset,[p, q] \cup[r, s]=[v, w]$, and

(2) $[p, q]$ and $[r, s]$ are both Boolean by induction assumption.

By (1), we may assume that $p=v$ and $s=w$ without loss of generality. Then

$$
\ell(q)=\ell(w)-1 \quad \text { and } \quad \ell(r)=\ell(v)+1 .
$$

Indeed, if $\ell(q) \leq \ell(w)-2$, then no element in $[v, q]$ is covered by $w$ while there are exactly $m-1$ elements in $[r, w]$ covered by $w$ because $[r, w]$ is Boolean and of rank $m-1$. Therefore, the number of coatoms in $[v, w]$ is $m-1$. On the other hand, since $Q_{v, w}$ is an $m$-cube, there must be $m$ coatoms in $[v, w]$. This is a contradiction. Therefore, $\ell(q)=\ell(w)-1$. A similar argument shows that $\ell(r)=\ell(v)+1$.

Since $Q_{v, q}$ is an $(m-1)$-cube, we have $\operatorname{dim} Q_{v, q}=\ell(q)-\ell(v)$ by induction assumption. Here $\ell(q)=\ell(w)-1$ by (5.3) and $\operatorname{dim} Q_{v, q}+1=\operatorname{dim} Q_{v, w}$ since $Q_{v, q}$ is a facet of $Q_{v, w}$. These show that $m=\operatorname{dim} Q_{v, w}=\ell(w)-\ell(v)$, i.e., $Q_{v, w}$ is toric.

We need to show that $[v, w]$ is Boolean. Since $Q_{v, w}$ is toric, any interval $[x, y] \subset[v, w]$ produces a face of $Q_{v, w}$ by Theorem 5.1. This in particular shows that the numbers of atoms and coatoms in $[v, w]$ are both $m=\ell(w)-\ell(v)$ since $Q_{v, w}$ is an $m$-cube. Moreover, since $Q_{v, w}$ is a cube, so is $Q_{x, y}$ and its dimension is $\ell(y)-\ell(x)$ as observed above. Therefore if $[x, y]$ is a proper subset of $[v, w]$, then one can apply the induction assumption to $Q_{x, y}$ so that $[x, y]$ is Boolean. In particular, for $u \in(v, w),[v, u]$ and $[u, w]$ are proper subsets of $[v, w]$ so that they are Boolean. This means that $u$ covers exactly $\ell(u)-\ell(v)$ elements and is covered by exactly $\ell(w)-\ell(u)$ elements. (This holds even when $u=v$ or $u=w$ as observed above.) This almost shows that $[v, w]$ is Boolean but we have to observe cover relations among elements to conclude that it is Boolean.

For $u \in[v, w]$ we set $\operatorname{rank} u=\ell(u)-\ell(v)$. As observed at the beginning of the proof of the "only if" part, there are elements $q, r \in[v, w]$ such that

(1) $\operatorname{rank} q=m-1, \operatorname{rank} r=1$, and

(2) $[v, q] \cap[r, w]=\emptyset,[v, q] \cup[r, w]=[v, w]$.

Since $[v, q]$ is Boolean and of rank $m-1$ and $\operatorname{rank} v=0$, we may regard $[v, q]$ as the Boolean algebra obtained from the set $[m-1]$. Then the atoms of $[v, w]$ are $\{1\},\{2\}, \ldots,\{m\}$. Since $\operatorname{rank} r=1$, we regard $r$ as $\{m\}$. Since $[r, w]$ is Boolean and of rank $m-1$ and $\operatorname{rank} r=1$, there are $m-1$ elements of rank 2 in $[r, w]$ and each of them covers $r(=\{m\})$ but since they are of rank 2, each of them must cover one more element which is in $[v, q]$. We denote by $\{i, m\}(i \in[m-1])$ the rank 2 element in $[r, w]$ which covers the atoms $\{i\}$ and $\{m\}$. Since $[r, w]$ is Boolean and of rank $m-1$, we may regard rank $k+1$ elements in $[r, w]$ as $\left\{i_{1}, \ldots, i_{k}, m\right\}$ where $\left\{i_{1}, \ldots, i_{k}\right\}$ is a subset of $[m-1]$ and $\left\{i_{1}, \ldots, i_{k}, m\right\}$ covers $\left\{i_{1}, \ldots, \widehat{i_{j}}, \ldots, i_{k}, m\right\}(1 \leq j \leq k)$.

The element $\left\{i_{1}, \ldots, i_{k}, m\right\}$ is of rank $k+1$ and already covers $k$ elements $\left\{i_{1}, \ldots, \widehat{i_{j}}, \ldots, i_{k}, m\right\}$ $(1 \leq j \leq k)$. Therefore, it suffices to show that

$$
\text { (*) } \quad\left\{i_{1}, \ldots, i_{k}, m\right\} \text { covers the element }\left\{i_{1}, \ldots, i_{k}\right\} \text { in }[v, q] \text {. }
$$

When $k=m-1$, the element $\left\{i_{1}, \ldots, i_{k}, m\right\}$ is the entire set $\{1, \ldots, m\}$ (that is $w$ ). In this case we already know that it covers all the coatoms of $[v, w]$. Therefore, we may assume $k<m-1$.

We shall prove $(*)$ above by induction on $k$. When $k=1,\left\{i_{1}, m\right\}$ covers $\left\{i_{1}\right\}$ by definition. Suppose that $(*)$ holds for $k-1$ and $2 \leq k<m-1$. We look at the interval $I$ between the empty set (that is $v$ ) and $\left\{i_{1}, \ldots, i_{k}, m\right\}$. Since $k<m-1, I$ is a proper subset of $[v, w]$; so $I$ is 
Boolean and we know that $\left\{i_{1}, \ldots, \hat{i_{j}}, \ldots, i_{k}, m\right\}(1 \leq j \leq k)$ are all in $I$. Therefore, $I$ contains all subsets of $\left\{i_{1}, \ldots, i_{k}, m\right\}$ except $\left\{i_{1}, \ldots, i_{k}\right\}$ which follows from induction assumption and the fact that $I$ is Boolean. The only missing element in $I$ lies in $[v, q]$ and is of rank $k$, i.e., of the form $\left\{j_{1}, \ldots, j_{k}\right\}$ where $\left\{j_{1}, \ldots, j_{k}\right\}$ is a subset of $[m-1]$. Here, $\left\{j_{1}, \ldots, j_{k}\right\}$ must contain all proper subsets of $\left\{i_{1}, \ldots, i_{k}\right\}$ since those proper subsets are in $I$ and $I$ is Boolean. Therefore, $\left\{j_{1}, \ldots, j_{k}\right\}=\left\{i_{1}, \ldots, i_{k}\right\}$. This completes the induction step and the proof of the proposition.

The following examples show that one cannot drop either toric or Boolean in Theorem 5.7 .

Example 5.9. (1) Let $v=1324$ and $w=4231$. Then $[v, w]$ is Boolean of length 4 but since $\operatorname{dim} Q_{v, w}=3, Q_{v, w}$ is not toric. The vertices $v$ and $w$ have degree 4 , so $Q_{v, w}$ is not a cube.

(2) Let $v=1324$ and $w=3412$. Then $v=s_{2}, w=v s_{3} s_{1} s_{2}=v s_{1} s_{3} s_{2}$, and $\operatorname{dim} Q_{v, w}=3$. Hence $Q_{v, w}$ is toric. But $[v, w]$ is a 4 -crown and not Boolean. The vertices $v$ and $w$ have degree 4 and the others are simple vertices, so $Q_{v, w}$ is not a cube.

Similarly, if $v=2143$ and $w=4231$, then $v=s_{1} s_{3}=s_{3} s_{1}, w=v s_{2} s_{3} s_{1}=v s_{2} s_{1} s_{3}$, and $\operatorname{dim} Q_{v, w}=3$. Hence $Q_{v, w}$ is toric but $[v, w]$ is a 4-crown and not Boolean.

It was shown in [19, Theorem 3.5.2] that the largest rank of Boolean Bruhat intervals in $\mathfrak{S}_{n+1}$ is at least $n+\left\lfloor\frac{n-1}{2}\right\rfloor$ by finding a sufficient condition on $v$ and $w$ for $[v, w]$ to be Boolean. This implies that there are many Boolean Bruhat intervals which are not toric like the Boolean interval $[1324,4231]$.

The following proposition geometrically means that a toric Richardson variety $X_{w}^{v}$ is smooth if it is smooth at either $v B$ or $w B$ :

Proposition 5.10. If $Q_{v, w}$ is toric and either $v$ or $w$ is a simple vertex, then $Q_{v, w}$ is simple. (Indeed, $Q_{v, w}$ is a cube by Proposition [5.6.)

Proof. We set $m=\ell(w)-\ell(v)$ and prove the proposition by induction on $m$. Suppose that the vertex $v$ is simple (the same argument works when the vertex $w$ is simple). We shall prove that $w$ is also a simple vertex. Let $y$ be a coatom in $[v, w]$. Since $Q_{v, w}$ is toric, $Q_{v, y}$ is a facet by Theorem 5.1 , Since $v$ is a simple vertex and $\operatorname{dim} Q_{v, w}=m$, there are exactly $m$ atoms and the direction vectors of the $m$ edges incident to $v$ are linearly independent. This means that $Q_{v, y}$ contains exactly $m-1$ edges incident to $v$ since $\operatorname{dim} Q_{v, y}=m-1$. Therefore, the number of coatoms in $[v, w]$ is at most $\left(\begin{array}{c}m \\ m-1\end{array}\right)=m$. However, since $\operatorname{dim} Q_{v, w}=m$, there must be at least $m$ coatoms. Therefore the number of coatoms in $[v, w]$ is $m$, which means that $w$ is a simple vertex.

Let $u \in(v, w)$. Since $Q_{v, w}$ is toric, both $Q_{v, u}$ and $Q_{u, w}$ are toric; so $\operatorname{dim} Q_{v, u}=\ell(u)-\ell(v)$ and $\operatorname{dim} Q_{u, w}=\ell(w)-\ell(u)$ and they are strictly less than $m$. As observed above, $v$ and $w$ are simple vertices of $Q_{v, w}$ and this means that they are also simple vertices of $Q_{v, u}$ and $Q_{u, w}$. Therefore one can apply the induction assumption to $Q_{v, u}$ and $Q_{u, w}$, so that they are both simple. These show that $u$ covers $\ell(u)-\ell(v)$ elements and is covered by $\ell(w)-\ell(u)$ elements. Hence $u$ is a simple vertex of $Q_{v, w}$, proving the proposition.

Example 5.9(2) shows that there is a toric Bruhat interval polytope $Q_{v, w}$ such that

$$
d(v)=d(w)=4>\operatorname{dim} Q_{v, w}=3 .
$$

Here is an example of a toric Bruhat interval polytope $Q_{v, w}$ with $d(v) \neq d(w)$.

Example 5.11. Let $v=13254$ and $w=35142$. Since $v=s_{2} s_{4}$ and $w=v s_{3} s_{4} s_{1} s_{2}$, we get $\ell(w)-\ell(v)=4$. It follows from (4.3) that

$$
\operatorname{dim} Q_{v, w}=5-\# B_{v, w}=4 .
$$

Hence the polytope $Q_{v, w}$ is toric. As we can see in Figure 10, there are six atoms and five coatoms. By Theorem [5.1, $d(v)=6$ and $d(w)=5$. We note that $Q_{v, w}$ is not a cube. 


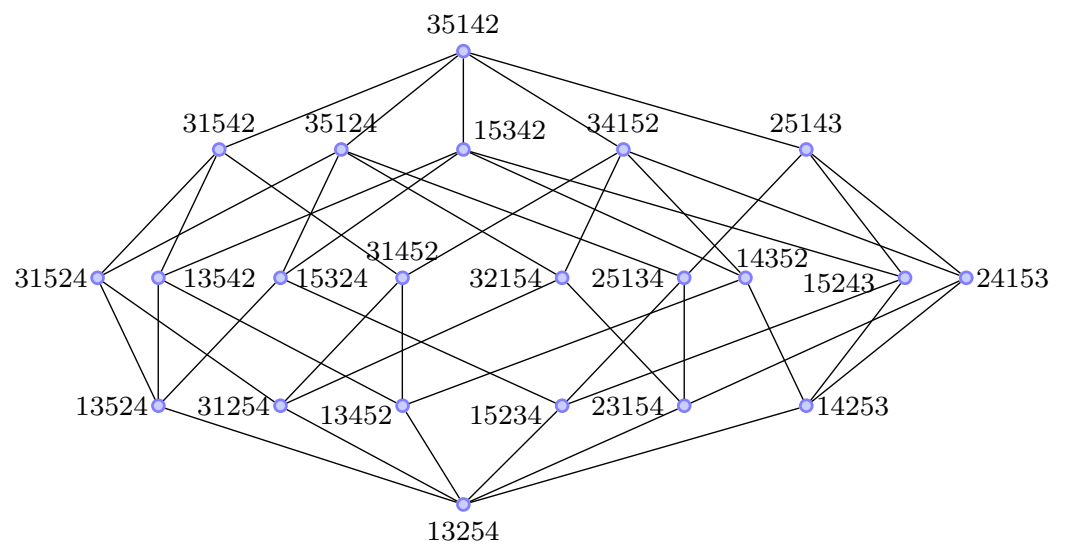

Figure 10. Bruhat interval [13254,35142].

We note that if $Q_{v, w}$ is a cube, then $[v, w]$ is Boolean and $Q_{v, w}$ is toric by Theorem 5.7 and $Q_{v, w}$ is obviously simple.

Corollary 5.12. Any two of the following three statements imply that $Q_{v, w}$ is a cube and hence imply the remaining one:

(1) $[v, w]$ is Boolean.

(2) $Q_{v, w}$ is toric.

(3) Either $v$ or $w$ is a simple vertex of $Q_{v, w}$.

Proof. By Theorem 5.7 and Proposition 5.10, it suffices to show that (1) and (3) imply (2). Suppose that (1) and (3) hold. Since $[v, w]$ is Boolean, the numbers of edges at $v$ and $w$ are equal to $\ell(w)-\ell(v)$. On the other hand, since either $v$ or $w$ is a simple vertex of $Q_{v, w}$, the numbers of edges at $v$ and $w$ are equal to $\operatorname{dim} Q_{v, w}$. Therefore $\operatorname{dim} Q_{v, w}=\ell(w)-\ell(v)$, that is, $Q_{v, w}$ is toric.

\section{Product of Bruhat intervals}

In this section, we will show that there are infinitely many non-simple toric Bruhat interval polytopes (see Proposition 6.4).

Let $r$ be a non-negative integer. To a pair $(x, y) \in \mathfrak{S}_{p} \times \mathfrak{S}_{q}$, we associate an element in $\mathfrak{S}_{p+q+r-1}$, denoted by $x *_{r} y$, as follows: express $x=s_{i_{1}} \cdots s_{i_{k}} \in \mathfrak{S}_{p}, y=s_{j_{1}} \cdots s_{j_{\ell}} \in \mathfrak{S}_{q}$, and define

$$
x *_{r} y:=\left(s_{i_{1}} \cdots s_{i_{k}}\right)\left(s_{j_{1}+p+r-1} \cdots s_{j_{\ell}+p+r-1}\right) \in \mathfrak{S}_{p+q+r-1} .
$$

Since $i_{1}, \ldots, i_{k}$ are less than or equal to $p-1$ while $j_{1}+p+r-1, \ldots, j_{\ell}+p+r-1$ are greater than or equal to $p, x *_{r} y$ is well-defined, that is, independent of the expressions of $x$ and $y$ above. The expressions of $x$ and $y$ need not be reduced but if they are reduced, then the resulting expression of $x *_{r} y$ in (6.1) is also reduced and hence

$$
\ell\left(x *_{r} y\right)=\ell(x)+\ell(y) .
$$

The following lemma would be obvious.

Lemma 6.1. Let $x, x^{\prime} \in \mathfrak{S}_{p}$ and $y, y^{\prime} \in \mathfrak{S}_{q}$. Then

(1) $x^{\prime} *_{r} y^{\prime} \leq x *_{r} y$ if and only if $x^{\prime} \leq x$ and $y^{\prime} \leq y$,

(2) $x^{\prime} *_{r} y^{\prime} \lessdot x *_{r} y$ if and only if $x^{\prime} \lessdot x$ and $y^{\prime}=y$ or $x^{\prime}=x$ and $y^{\prime} \lessdot y$. 
Moreover, if $z \leq x *_{r} y$, then $z=x^{\prime} *_{r} y^{\prime}$ for some $x^{\prime} \leq x$ and $y^{\prime} \leq y$.

Suppose that $x^{\prime} \leq x$ and $y^{\prime} \leq y$. Then it follows from Lemma 6.1 that

$$
\left[x^{\prime} *_{r} y^{\prime}, x *_{r} y\right]=\left\{a *_{r} b \mid x^{\prime} \leq a \leq x, y^{\prime} \leq b \leq y\right\} .
$$

One can also see that

$$
\operatorname{dim} Q_{x^{\prime} *_{r} y^{\prime}, x *_{r} y}=\operatorname{dim} Q_{x^{\prime}, y^{\prime}}+\operatorname{dim} Q_{x, y}
$$

using Theorem 4.6 in [22]. Therefore, we have

Corollary 6.2. The poset structure of $\left[x^{\prime} *_{r} y^{\prime}, x *_{r} y\right]$ is independent of $r(r \geq 0)$. Moreover, $\left[x^{\prime} *_{r} y^{\prime}, x *_{r} y\right]$ is Boolean if and only if both $\left[x^{\prime}, x\right]$ and $\left[y^{\prime}, y\right]$ are Boolean, and $Q_{x^{\prime} *_{r} y^{\prime}, x *_{r} y}$ is toric if and only if both $Q_{x^{\prime}, x}$ and $Q_{y^{\prime}, y}$ are toric.

We set

$$
c(v, w):=\ell(w)-\ell(v)-\operatorname{dim} Q_{v, w}
$$

and call it the complexity of the interval $[v, w]$ because it is the complexity of the torus action on the Richardson variety $X_{w}^{v}$. By (3.4), we get $c(v, w) \geq 0$ and the equality holds when $Q_{v, w}$ is toric by definition. It follows from (6.2) and (6.3) that

$$
c\left(x^{\prime} *_{r} y^{\prime}, x *_{r} y\right)=c\left(x^{\prime}, y^{\prime}\right)+c(x, y) .
$$

Example 6.3. (1) For $v=1324$ and $w=4231$ in Example 5.9(1), we get that

$$
c(v, w)=\ell(w)-\ell(v)-\operatorname{dim} Q_{v, w}=5-1-3=1 .
$$

Moreover, $[v, w]$ is a Boolean interval of length 4 .

(2) For $v=1324$ and $w=3412$ in Example 5.9 (2), the Bruhat interval polytope $Q_{v, w}$ is toric of dimension 3 , and we have that

$$
d(v)=d(w)=4=\operatorname{dim} Q_{v, w}+1 .
$$

(3) For $v=13254$ and $w=35142$ in Example 5.11, the Bruhat interval polytope $Q_{v, w}$ is toric of dimension 4 , and we see that

$$
|d(v)-d(w)|=|6-5|=1 .
$$

The following implies that there are infinitely many toric singular Bruhat interval polytopes.

Proposition 6.4. For any non-negative integer $k$,

(1) there is a Boolean interval $[v, w]$ with $c(v, w)=k$,

(2) there is a toric Bruhat interval polytope $Q_{v, w}$ such that $d(v)=d(w)=\operatorname{dim} Q_{v, w}+k$, and

(3) there is a toric Bruhat interval polytope $Q_{v, w}$ with $|d(v)-d(w)|=k$.

Proof. The case $k=0$ is realized by a cube $Q_{v, w}$, so we may assume $k \geq 1$. The complexity $c(v, w)$, the degrees $d(v)$ and $d(w)$, and $\operatorname{dim} Q_{v, w}$ behave additively with respect to the product $*_{r}$ of a copy of $[v, w]$, so each statement respectively follows from Example 6.3 .

\section{Conditions on $v$ And $w$ FOR $Q_{v, w}$ TO Be tORIC}

In this section, we first find some sufficient conditions on $v$ and $w$ for $Q_{v, w}$ to be toric, and then find a sufficient condition for such a toric Bruhat interval polytope $Q_{v, w}$ to be a cube.

It was shown in [11, $\S 5$ and $\S 6]$ that $Q_{v, w}$ is toric (in fact, a cube) if $v=\left[a_{1}, \ldots, a_{n-1}, n\right]$ and $w=\left[n, a_{1}, \ldots, a_{n-1}\right]$ or $v=\left[1, b_{2}, \ldots, b_{n}\right]$ and $w=\left[b_{2}, \ldots, b_{n}, 1\right]$. In these cases,

$$
\begin{aligned}
& w=v s_{n-1} s_{n-2} \cdots s_{1} \quad \text { and } \quad \ell(w)-\ell(v)=n-1, \\
& w=v s_{1} s_{2} \cdots s_{n-1} \quad \text { and } \quad \ell(w)-\ell(v)=n-1 .
\end{aligned}
$$


These examples motivate us to study the following case:

$$
w=v s_{j_{1}} s_{j_{2}} \cdots s_{j_{m}} \text { where } \ell(w)-\ell(v)=m \text { and } j_{1}, \ldots, j_{m} \text { are distinct. }
$$

Proposition 7.1. Suppose that $w=v s_{j_{1}} s_{j_{2}} \cdots s_{j_{m}}$ or $w=s_{j_{1}} s_{j_{2}} \cdots s_{j_{m}} v$ with $\ell(w)-\ell(v)=m$. Then $j_{1}, \ldots, j_{m}$ are distinct if and only if $Q_{v, w}$ is toric.

Proof. Since $w=s_{j_{1}} s_{j_{2}} \cdots s_{j_{m}} v$ means $w^{-1}=v^{-1} s_{j_{1}} s_{j_{2}} \cdots s_{j_{m}}$, it is enough to prove when $w=$ $v s_{j_{1}} s_{j_{2}} \cdots s_{j_{m}}$ by Corollary [5.3. Since $m=\ell(w)-\ell(v)$, we have $\ell\left(v s_{j_{1}} s_{j_{2}} \cdots s_{j_{k}}\right)=\ell(v)+k$ for any $1 \leq k \leq m$. This means that

$$
v \lessdot v s_{j_{1}} \lessdot v s_{j_{1}} s_{j_{2}} \lessdot \cdots \lessdot v s_{j_{1}} s_{j_{2}} \cdots s_{j_{m}}=w
$$

is a maximal chain from $v$ to $w$, say $\mathcal{C}$. Then it defines the graph $G^{\mathbb{C}}$ whose edge set is given by $\left\{\left\{j_{1}, j_{1}+1\right\}, \ldots,\left\{j_{m}, j_{m}+1\right\}\right\}$ (see Section 4). Hence the number of connected components of $G^{\mathcal{C}}$ is greater than or equal to $n-m$. Thus the dimension of $Q_{v, w}$ is less than or equal to $m$ by (4.3). Notice that $G^{\mathcal{E}}$ has exactly $n-m$ components if and only if $j_{1}, j_{2}, \ldots, j_{m}$ are distinct. Hence $Q_{v, w}$ is toric if and only if $j_{1}, j_{2}, \ldots, j_{m}$ are distinct.

Example $5.9(2)\left(w=v s_{3} s_{1} s_{2}=v s_{1} s_{3} s_{2}\right.$ where $\left.v=s_{2}\right)$ and Example $5.11\left(w=v s_{3} s_{4} s_{1} s_{2}\right.$ where $v=s_{2} s_{4}$ ) show that we cannot conclude that $Q_{v, w}$ is a cube in Proposition 7.1. We shall give a sufficient condition on $v$ and $w$ for $Q_{v, w}$ to be a cube. For that we prepare some notations. For $p$ and $q$ in $[n-1]$, we set

$$
s(p, q)= \begin{cases}s_{p} s_{p+1} \cdots s_{q} & \text { when } p \leq q, \\ s_{p} s_{p-1} \cdots s_{q} & \text { when } p \geq q .\end{cases}
$$

For each $s(p, q)$, we also set

$$
\bar{p}=\min \{p, q\}, \quad \bar{q}=\max \{p, q\} .
$$

We note that if $j_{1}, \ldots, j_{m} \in[n]$ are distinct, then we have a minimal expression

$$
s_{j_{1}} s_{j_{2}} \cdots s_{j_{m}}=s\left(p_{1}, q_{1}\right) s\left(p_{2}, q_{2}\right) \cdots s\left(p_{r}, q_{r}\right)
$$

where the intervals $\left[\bar{p}_{1}, \bar{q}_{1}\right], \ldots,\left[\bar{p}_{r}, \bar{q}_{r}\right]$ are disjoint and $r$ is the minimum among such expressions.

Example 7.2. Here are examples of minimal expressions.

(1) $s_{1} s_{2} \cdots s_{n-1}=s(1, n-1), \quad s_{n-1} s_{n-2} \cdots s_{1}=s(n-1,1)$.

(2) $s_{1} s_{3} s_{8} s_{2} s_{4} s_{7} s_{6}=s_{3} s_{4} s_{1} s_{2} s_{8} s_{7} s_{6}=s(3,4) s(1,2) s(8,6)$.

(3) $s_{2} s_{8} s_{4} s_{7} s_{1} s_{6}=s_{2} s_{1} s_{4} s_{8} s_{7} s_{6}=s(2,1) s(4,4) s(8,6)$.

We say that the product $s_{j_{1}} s_{j_{2}} \cdots s_{j_{m}}$ in (7.2) is proper if no two intervals among $\left[\bar{p}_{1}, \bar{q}_{1}\right], \ldots,\left[\bar{p}_{r}, \bar{q}_{r}\right]$ are adjacent, in other words, the cycles defined by $s\left(p_{1}, q_{1}\right), \ldots, s\left(p_{r}, q_{r}\right)$ are disjoint. For instance, (1) and (3) in Example 7.2 are proper but (2) is not because the intervals [3,4] and [1,2] are adjacent.

Proposition 7.3. Suppose that $s_{j_{1}} s_{j_{2}} \cdots s_{j_{m}}$ is a proper minimal expression. If $w=v s_{j_{1}} s_{j_{2}} \cdots s_{j_{m}}$ or $w=s_{j_{1}} s_{j_{2}} \cdots s_{j_{m}} v$ with $\ell(w)-\ell(v)=m$, then the Bruhat interval polytope $Q_{v, w}$ is a cube.

Proof. Since $w=s_{j_{1}} s_{j_{2}} \cdots s_{j_{m}} v$ means $w^{-1}=v^{-1} s_{j_{1}} s_{j_{2}} \cdots s_{j_{m}}$, it is enough to prove the proposition when $w=v s_{j_{1}} s_{j_{2}} \cdots s_{j_{m}}$ by Corollary 5.8. We know that $Q_{v, w}$ is toric by Proposition 7.1 and hence every cover relation in $[v, w]$ gives an edge in $Q_{v, w}$ by Theorem 5.1. Thus it suffices to show that $v$ is covered by exactly $\ell(w)-\ell(v)$ elements in $[v, w]$ by Proposition 5.10 .

Note that when $w=v s(1, n-1)$ or $v s(n-1,1)$, we know that $Q_{v, w}$ is an $(n-1)$-cube by [11, §5]. Therefore $v$ is covered by exactly $(n-1)$ elements in $[v, w]$ (we will show how to find those $(n-1)$ elements after the proof of the proposition). 
Now we assume that $w=v s\left(p_{1}, q_{1}\right) \cdots s\left(p_{r}, q_{r}\right)$, where $s\left(p_{1}, q_{1}\right) \cdots s\left(p_{r}, q_{r}\right)$ is a minimal expression with $r \geq 2$. For $u \in \mathfrak{S}_{n}$ and $1 \leq a<b \leq n$, we denote the block from $u(a)$ to $u(b)$ in the one-line notation of $u$ by $u([a, b])$. Since no two intervals among $\left[\bar{p}_{1}, \bar{q}_{1}\right], \ldots,\left[\bar{p}_{r}, \bar{q}_{r}\right]$ are adjacent, we have

$$
w\left(\left[\bar{p}_{i}, \bar{q}_{i}+1\right]\right)=v\left(\left[\bar{p}_{i}, \bar{q}_{i}+1\right]\right) s\left(p_{i}, q_{i}\right) \quad \text { for each } i=1,2, \ldots, r .
$$

Namely, on each block $\left[\bar{p}_{i}, \bar{q}_{i}+1\right]$, the situation is the same as the first case treated above. Therefore, $v$ is covered by exactly $\sum_{i=1}^{r}\left(\bar{q}_{i}+1-\bar{p}_{i}\right)$ elements in $[v, w]$. Here, $\sum_{i=1}^{r}\left(\bar{q}_{i}+1-\bar{p}_{i}\right)=\ell(w)-\ell(v)$, so this proves the proposition. To be more precise, we need the following observation. Any cover relation is obtained by a right transposition and we need to see that any right transposition on $v$ which gives a cover relation in $[v, w]$ is a transposition on some block $\left[\bar{p}_{i}, \bar{q}_{i}+1\right]$. But this is true because the partition $B_{v, w}$ of $[n]$ is given by

$$
B_{v, w}=\bigcup_{i=1}^{r}\left[\bar{p}_{i}, \bar{q}_{i}+1\right] \cup \bigcup_{k \in[n] \backslash \bigcup_{i=1}^{r}\left[\bar{p}_{i}, \bar{q}_{i}+1\right]}\{k\}
$$

and a right transposition on $v$ which gives a cover relation in $[v, w]$ must preserve the partition $B_{v, w}$ since the partition $B_{v, w}$ is independent of a choice of maximal chains from $v$ to $w$ by [22, Corollary 4.8].

We briefly explain how to find the atoms of $[v, w]$ when $w=v s(1, n-1)$ or $v s(n-1,1)$ (see the proof of Proposition 8.3). When $w=v s(1, n-1)$ and $\ell(w)-\ell(v)=n-1$ (this means that $v(1)=1$ ). We write $v=v(1) v(2) \ldots v(n)$ in one-line notation where $v(1)=1$. For each $v(j)$ for $2 \leq j \leq n$, we find $v(i)$ such that $i<j, v(i)<v(j)$ and $v(k)>v(j)$ for any $i<k<j$, and interchange $v(i)$ and $v(j)$. The resulting element covers $v$ and one can check that it is in $[v, w]$. The $(n-1)$ elements obtained in this way are the desired elements.

When $w=v s(n-1,1)$ and $\ell(w)-\ell(v)=n-1$ (this means that $v(n)=n$ ), the method to find elements in $[v, w]$ which cover $v$ is essentially same as above. We write $v=v(1) v(2) \ldots v(n)$ in one-line notation where $v(n)=n$. For each $v(i)$ for $1 \leq i \leq n-1$, we find $v(j)$ such that $i<j$, $v(i)<v(j)$ and $v(i)>v(k)$ for any $i<k<j$ and interchange $v(i)$ and $v(j)$. The resulting element covers $v$ and one can check that it is in $[v, w]$. The $(n-1)$ elements obtained in this way are the desired elements.

Example 7.4. (1) Take $v=14325$ and $w=v s(1,4)=43251$. In this case, the four elements in $[v, w]$ which cover $v$ are

$$
41325, \quad 34125, \quad 24315, \quad 14352 .
$$

Although 15324 and 14523 cover $v$, they are not in $[v, w]$.

(2) Take the same $v=14325$ as above but $w=v s(4,1)=51432$. In this case, the four elements in $[v, w]$ which cover $v$ are

$$
41325, \quad 15324, \quad 14523, \quad 14352 .
$$

Although 34125 and 24315 cover $v$, they are not in $[v, w]$.

(3) Take the same $v=14325$ as above but $w=v s(1) s(4,3)=41532$. There are three elements in $[v, w]$ cover $v$ :

$$
41325=14325(1,2), \quad 14523=14325(3,5), \quad 14352=14325(4,5) .
$$

We have that $\{1,2\} \subset[1,2]$ and $\{3,5\},\{4,5\} \subset[3,5]$. Although $34125=14325(1,3)$, $24315=14325(1,4)$, and $15324=14325(2,5)$ which cover $v$, they are not in $[v, w]$. Here, one can see that none of the subsets $\{1,3\},\{1,4\},\{2,5\}$ of $[5]$ are contained in $[1,2]$ or $[3,5]$. 
Now we consider the converse of Proposition 7.3. If the product in (7.2) is not proper, then we may assume that the intervals $\left[\bar{p}_{1}, \bar{q}_{1}\right]$ and $\left[\bar{p}_{2}, \bar{q}_{2}\right]$ in (7.2) are adjacent by interchanging commuting factors if necessary. We set $(a, b)=\left(p_{1}, q_{1}\right)$ and $(c, d)=\left(p_{2}, q_{2}\right)$. Then

$$
w=v s(a, b) s(c, d) x \quad \text { where } \quad x=s\left(p_{3}, q_{3}\right) \cdots s\left(p_{r}, q_{r}\right) .
$$

The automorphism of $\mathfrak{S}_{n}$ given by conjugation by $w_{0}$ maps $s_{i}$ to $s_{n-i}$ and hence maps $s(a, b) s(c, d)$ to $s(n-a, n-b) s(n-c, n-d)$, so we may assume $a \leq b$ without loss of generality.

Lemma 7.5. Assume that $a \leq b$ and $s(a, b) s(c, d)$ is minimal but not proper. Then the following three cases occur:

(1) $b+1=d, c>d$,

(2) $a-1=c, c \geq d, a<b$,

(3) $a-1=d, c<d$.

Proof. Since the intervals $[a, b]$ and $[\bar{c}, \bar{d}]$ are adjacent, we have $b+1=\bar{c}$ or $a-1=\bar{d}$. Then there are four possible cases.

(0) If $b+1=\bar{c}=c$, then $c \leq d$ and hence $s(a, b) s(c, d)=s(a, d)$ which contradicts the minimality of the expression $s(a, b) s(c, d) x$. Thus the case $b+1=c$ does not occur.

(1) If $b+1=\bar{c}=d$, then $c \geq d$ but the case $c=d$ is excluded above. Hence $c>d$ which is case (1).

(2) If $a-1=\bar{d}=c$, then $c \geq d$. Moreover, if $a=b$, then $s(a, b) s(c, d)=s(a, d)$ which contradicts the minimality of the expression $s(a, b) s(c, d) x$. Thus we obtain case (2).

(3) If $a-1=\bar{d}=d$, then $c \leq d$. Here the case $c=d$ is included in case (2), so we obtain case (3).

Therefore, the only three cases occurs.

The following lemma shows that if a minimal expression $s(a, b) s(c, d)$ is not proper, then there exists a permutation $v$ such that $Q_{v, v s(a, b) s(c, d)}$ is not a cube.

Lemma 7.6. Let $v=s_{d}$ for cases (1) and (3) in Lemma 7.5 and $v=s(a+1, b) s_{c}$ for case (2) in Lemma 7.5. Then the Bruhat interval $[v, w]$ for $w$ in (7.3) has $\ell(w)-\ell(v)+1$ coatoms $\left(\right.$ so $Q_{v, w}$ is not a cube although it is toric).

Proof. We note that any coatom of $[v, w]$ is obtained by removing an element from a reduced expression of $w$ such that the resulting expression is reduced and contains a reduced expression of $v$.

For case (1) in Lemma 7.5 we have the reduced expression of $w$ :

$$
w=v s(a, b) s(c, d) x=s_{d}\left(s_{a} s_{a+1} \cdots s_{b-1} s_{b}\right)\left(s_{c} s_{c-1} \cdots s_{d+1} s_{d}\right) x .
$$

As remarked above, any coatom is obtained by removing an element from (7.4). In (7.4), only $s_{d}$ appears twice, the others appear only once, and the elements $s_{b}=s_{d-1}$ and $s_{d+1}$ which do not commute with $s_{d}$ appear between the two $s_{d}$ 's in (7.4). Noting these, one can see that removing any element from (7.4) produces a reduced expression and the resulting expression contains $v=s_{d}$. Therefore $[v, w]$ has exactly $\ell(w)-\ell(v)+1$ coatoms.

A similar argument works for case (3). In this case we have

$$
w=v s(a, b) s(c, d) x=s_{d}\left(s_{a} s_{a+1} \cdots s_{b-1} s_{b}\right)\left(s_{c} s_{c+1} \cdots s_{d-1} s_{d}\right) x
$$

and only $s_{d}$ appears twice and the elements $s_{a}=s_{d+1}$ and $s_{d-1}$ which do not commute with $s_{d}$ appear between the two $s_{d}$ 's. Therefore, removing any element from (7.5) produces a reduced expression and the resulting expression contains $v=s_{d}$, so $[v, w]$ has exactly $\ell(w)-\ell(v)+1$ coatoms. 
As for case (2), the situation is slightly different from the above two cases. In case (2) we have

$$
\begin{aligned}
w & =v s(a, b) s(c, d) x \\
& =\left(s_{a+1} s_{a+2} \cdots s_{b-1} s_{b} s_{c}\right)\left(\underline{s_{a} s_{a+1} s_{a+2} \cdots s_{b-1}} s_{b}\right)\left(s_{c} s_{c-1} \cdots s_{d+1} s_{d}\right) x .
\end{aligned}
$$

In this case, $s_{a+1}, s_{a+2}, \ldots, s_{b-1}, s_{b}, s_{c}\left(=s_{a-1}\right)$ appear twice and the others appear once in (7.6). One can see that removing any element from the underlined product in (7.6) does not produce a reduced expression. For instance, if we remove $s_{a}$, then $s_{c}$ commute with all the elements between the two $s_{c}$ in (7.6); so the resulting expression is not reduced. If we remove $s_{a+1}$ in the underlined product, then (17.6) turns into

$$
s_{c} s_{a+1} s_{a}\left(s_{a+2} \cdots s_{b-1} s_{b}\right)\left(s_{a+2} \cdots s_{b-1} s_{b}\right)\left(s_{c} s_{c-1} \cdots s_{d+1} s_{d}\right) x
$$

since $s_{c}\left(=s_{a-1}\right)$ commutes with all the elements $s_{k}$ for $a+1 \leq k \leq b$ and $s_{a}$ commutes with all the elements $s_{\ell}$ for $a+2 \leq \ell \leq b$. The above expression is not reduced because the product $\left(s_{a+2} \cdots s_{b-1} s_{b}\right)\left(s_{a+2} \cdots s_{b-1} s_{b}\right)$ can be reduced. A similar observation applies when we remove one of the other elements in the underlined product in (7.6).

On the other hand, removing an element not in the underlined product produces a reduced expression (for that the existence of $s_{a}$ in (7.6) is important). One can also see that the elements obtained in this way contains the reduced expression $s_{a+1} s_{a+2} \cdots s_{b-1} s_{b} s_{c}$ of $v$ and there are $\ell(w)-$ $\ell(v)+1$ number of such elements, so $[v, w]$ has exactly $\ell(w)-\ell(v)+1$ coatoms in this case too.

Proposition 7.3 and the two lemmas above say that the converse of Proposition 7.3 is true.

Corollary 7.7. The Bruhat interval polytope $Q_{v, w}$ is a cube for any $v$ and $w$ in (7.1) if and only if the product $s_{j_{1}} s_{j_{2}} \cdots s_{j_{m}}$ is proper.

Example 7.8. Suppose that $s_{j_{1}} s_{j_{2}} s_{j_{3}}=s_{3} s_{1} s_{2}$. Then the Bruhat interval polytope $Q_{v, w}$ is a cube when $v=e$ and $w=s_{3} s_{1} s_{2}$ since $s_{3}, s_{1}, s_{2}$ are distinct. On the other hand, for $v=s_{2}$ and $w=s_{2} s_{3} s_{1} s_{2}$, the Bruhat interval polytope $Q_{v, w}$ is not a cube by Example 5.9 (2). Indeed, the product $s_{3} s_{1} s_{2}=s(3,3) s(1,2)$ is not proper.

Below is another sufficient condition on $v$ and $w$ for $Q_{v, w}$ to be a cube.

Proposition 7.9. Suppose that $w$ is a product of distinct simple reflections (equivalently, $w$ avoiding the patterns 3412 and 321 by Tenner [20]). Then $Q_{v, w}$ is a cube for any $v$ and $w$ such that $v<w$. In particular, if $v$ and $w$ are as in (7.1) and $v$ has a reduced expression $s_{i_{1}} s_{i_{2}} \cdots s_{i_{\ell}}$ such that $i_{1}, \ldots, i_{\ell}, j_{1}, \ldots, j_{m}$ are all distinct, then $Q_{v, w}$ is a cube.

Proof. If $w$ is a product of distinct simple reflections, then $Q_{e, w}$ is a cube. Since $Q_{e, w}$ is in particular toric, $Q_{v, w}$ is a face of $Q_{e, w}$ for any $v$ such that $v<w$ by Theorem 5.1. Therefore $Q_{v, w}$ is also a cube.

So far, we have studied whether $Q_{v, w}$ is toric when there exist reduced expressions $r(v)$ and $r(w)$ for $v$ and $w$ such that the subword $r(w) \backslash r(v)$ of $r(w)$ is a product of distinct simple transpositions. Unfortunately, there is an example that $Q_{v, w}$ is a cube even though there are no reduced expressions for $v$ and $w$ such that $r(w) \backslash r(v)$ is distinct.

Example 7.10. Let $v=1243$ and $w=3412$. Then $v=s_{3}$ and $w=s_{2} s_{3} s_{1} s_{2}=s_{2} s_{1} s_{3} s_{2}$. Hence there are no reduced expressions of $v$ and $w$ such that $r(w) \backslash r(v)$ is a product of distinct simple transpositions. But one can check that the Bruhat interval polytope $Q_{v, w}$ is a 3-cube.

Therefore, it seems difficult to characterize $v$ and $w$ for which $Q_{v, w}$ is toric or combinatorially equivalent to a cube. 


\section{Finding All COATOMS IN SOME SPECIAL CASES}

In this section, we will find all coatoms in some special cases. We first find a necessary and sufficient condition for $w(i, j)$ to be a coatom of $[v, w]$ when $w=v s(1, n-1)$, and then conclude that there are exactly $(n-1)$ elements in $[v, v s(1, n-1)]$ without using the result in [11, §5]. After that, we consider the case $w=v s(a, b) s(c, d)$ where $s(a, b) s(c, d)$ is minimal. We give a necessary and sufficient condition for $w(i, j)$ to be a coatom of $[v, w]$ and then describe when $Q_{v, w}$ is a cube. Note that finding atoms is essentially same as finding coatoms because multiplication by the longest element $w_{0}$ reverses the Bruhat order.

The following lemma is obvious but plays a role in our argument.

Lemma 8.1. Let $c_{1}, \ldots, c_{d}$ be distinct positive integers.

(1) Let $a$ and $b$ be positive integers different from any $c_{i}$. Then

$$
\left\{c_{1}, \ldots, c_{d}, a\right\} \uparrow \leq\left\{c_{1}, \ldots, c_{d}, b\right\} \uparrow \quad \Longleftrightarrow \quad a \leq b .
$$

(2) Let $a_{1}$ and $a_{2}\left(\right.$ also $\left.b_{1}, b_{2}\right)$ be distinct positive integers different from any $c_{i}$. Then

$$
\left\{c_{1}, \ldots, c_{d}, a_{1}, a_{2}\right\} \uparrow \leq\left\{c_{1}, \ldots, c_{d}, b_{1}, b_{2}\right\} \uparrow \quad \Longleftrightarrow \quad\left\{a_{1}, a_{2}\right\} \uparrow \leq\left\{b_{1}, b_{2}\right\} \uparrow .
$$

Note that if $\left\{a_{1}, a_{2}\right\} \cap\left\{b_{1}, b_{2}\right\} \neq \emptyset$, then (2) reduces to (1) in the lemma above. We prepare one more lemma.

Lemma 8.2. Let $v \leq w$ and let $t_{i, j}(1 \leq i<j \leq n)$ be a transposition. Then $v \leq w t_{i, j}$ if and only if

$$
\{v(1), v(2), \ldots, v(p)\} \uparrow \leq\left\{w t_{i, j}(1), w t_{i, j}(2), \ldots, w t_{i, j}(p)\right\} \uparrow \text { for every } i \leq p<j .
$$

Proof. The condition $v \leq w t_{i, j}$ is equivalent to

$$
\{v(1), v(2), \ldots, v(p)\} \uparrow \leq\left\{w t_{i, j}(1), w t_{i, j}(2), \ldots, w t_{i, j}(p)\right\} \uparrow \text { for every } 1 \leq p<n .
$$

For $p<i$ or $p \geq j$, we have

$$
\left\{w t_{i, j}(1), w t_{i, j}(2), \ldots, w t_{i, j}(p)\right\}=\{w(1), w(2), \ldots, w(p)\} .
$$

Since $v \leq w$, this shows that the inequality above holds for $p<i$ or $p \geq j$, proving the lemma.

We set

$$
V(p)=\{v(1), \ldots, v(p)\}, \quad W_{i, j}(p)=\left\{w t_{i, j}(1), \ldots, w t_{i, j}(p)\right\} .
$$

We also introduce the following notation: if $A$ and $B$ are sets and $C$ is a subset of $A \cap B$, then we write

$$
(A, B) \equiv(A \backslash C, B \backslash C) .
$$

We will apply this notation to $V(p)$ and $W_{i, j}(p)$ later.

In this section we investigate the following case

$$
w=v s_{1} s_{2} \cdots s_{n-1} \quad \text { equivalently } \quad v=w s_{n-1} \cdots s_{2} s_{1}
$$

where $\ell(w)-\ell(v)=n-1$. (A similar argument works when $w=v s_{n-1} s_{n-2} \cdots s_{1}$.) Since $\ell(w)-$ $\ell(v)=n-1$, it follows from (8.1) that

$$
w(n)<w(1), \ldots, w(n-1) .
$$

Note that these inequalities imply that $w(n)=1$.

Proposition 8.3. Let $v$ and $w$ be as in (8.1). Then $w t_{i, j}$ is a coatom of $[v, w]$ if and only if $w(i)>w(j)$ and $w(p)>w(i)$ for every $i<p<j$. Moreover, there are exactly $n-1$ coatoms in $[v, w]$ and hence $Q_{v, w}$ is a cube. 
Proof. We note that $\ell\left(w t_{i, j}\right)=\ell(w)-1$ if and only if $w(i)>w(j)$ and $w(p) \notin[w(j), w(i)]$ for every $i<p<j$. We shall show that under this situation, the condition $v \leq w t_{i, j}$ is equivalent to the condition $w(i)<w(p)$ for every $i<p<j$.

It follows from (8.1) that we have

$$
\begin{aligned}
& w t_{i, j}=w(1) w(2) \ldots w(i-1) \quad w(j) \quad w(i+1) \ldots w(j-1) \quad w(i) \quad w(j+1) \ldots \quad w(n), \\
& v=w(n) w(1) \ldots w(i-2) w(i-1) \quad w(i) \quad \ldots w(j-2) w(j-1) \quad w(j) \quad \ldots w(n-1) .
\end{aligned}
$$

Therefore, we have

$$
\left(V(p), W_{i, j}(p)\right) \equiv \begin{cases}(\{w(n)\},\{w(j)\}) & \text { for } p=i \\ (\{w(n), w(i)\},\{w(j), w(p)\}) & \text { for } i<p<j\end{cases}
$$

Then

$$
\begin{aligned}
& V(p) \uparrow \leq W_{i, j}(p) \uparrow \Longleftrightarrow w(n) \leq w(j) \quad \text { for } p=i, \\
& V(p) \uparrow \leq W_{i, j}(p) \uparrow \Longleftrightarrow\{w(n), w(i)\} \uparrow \leq\{w(j), w(p)\} \uparrow \quad \text { for } i<p<j
\end{aligned}
$$

by Lemma 8.1. Since $1=w(n)<w(j)<w(i)$, it follows from Lemma 8.2 that $w t_{i, j}$ is a coatom of $[v, w]$ if and only if $w(i)<w(p)$ for every $i<p<j$, proving the former statement of the proposition.

For each $1 \leq i<n$, there exists $j$ satisfying the condition in the former statement since $w(n)=1$, and such $j$ is unique for each $i$. This proves the latter statement in the proposition.

In the remainder of this section we will treat the case

$$
w=v s(a, b) s(c, d) \quad(s(a, b) s(c, d) \neq s(\bar{a}, \bar{d})),
$$

where $[\bar{a}, \bar{b}] \cup[\bar{c}, \bar{d}]=[1, n-1]$ and $\ell(w)-\ell(v)=n-1$. The conjugation on $\mathfrak{S}_{n}$ by $w_{0}$ maps $s_{i}$ to $s_{n-i}$, so it suffices to consider the case where $a<b$. There are three cases:

$\mathrm{I}:(a, b)=(1, k-1),(c, d)=(n-1, k)$,

II : $(a, b)=(k, n-1),(c, d)=(k-1,1)$,

III : $(a, b)=(k, n-1),(c, d)=(1, k-1)$,

where $n \geq 4$ and $2 \leq k \leq n-2$.

In the following, we assume that $\ell\left(w t_{i, j}\right)=\ell(w)-1$, so

$$
w(i)>w(j) \text { and } w(p) \notin[w(j), w(i)] \text { for every } i<p<j .
$$

We keep in mind that $w(i)>w(j)$ throughout this section unless otherwise stated. We shall observe that the condition $v \leq w t_{i, j}$ gives stronger conditions than the above. By Lemma 8.2 , it suffices to check

$$
V(p) \uparrow \leq W_{i, j}(p) \uparrow \quad \text { for } i \leq p<j .
$$

Case I. In this case we have

$$
\begin{aligned}
w & =v\left(s_{1} \cdots s_{k-1}\right)\left(s_{n-1} \cdots s_{k}\right), \quad \text { equivalently } \\
v & =w\left(s_{k} \cdots s_{n-1}\right)\left(s_{k-1} \cdots s_{1}\right) .
\end{aligned}
$$

Since $\ell(w)-\ell(v)=n-1$, we have

$$
\begin{aligned}
& w(k+1), w(k+2), \ldots, w(n)<w(k), \\
& w(k+1)<w(1), \ldots, w(k-1) .
\end{aligned}
$$

Proposition 8.4. In Case I, $w t_{i, j}$ is a coatom of $[v, w]$ if and only if one of the following is satisfied:

(1) If $1 \leq i<j \leq k+1$, then $w(i)<w(p)$ for every $i<p<j$.

(2) If $k \leq i<j \leq n$, then $w(p)<w(j)$ for every $i<p<j$. 
(3) If $1 \leq i<k$ and $k+1<j \leq n$, then $w(i)<w(p)$ for every $i<p \leq k$ and $w(p)<w(j)$ for every $k<p<j$.

Moreover, $Q_{v, w}$ is a cube in Case I if and only if there is no pair $(i, j)$ in (3).

Proof. (1) In this case we have

$$
\begin{aligned}
& w t_{i, j}=w(1) \quad w(2) \ldots w(i-1) \quad w(j) \quad w(i+1) \ldots w(j-1) \quad w(i) \quad \ldots \quad w(k) \quad w(k+1) \ldots w(n-1) w(n), \\
& v=w(k+1) w(1) \ldots w(i-2) \quad w(i-1) \quad w(i) \quad \ldots w(j-2) w(j-1) \ldots w(k-1) w(k+2) \ldots \quad w(n) \quad w(k) .
\end{aligned}
$$

where $w(i-1)$ in the line of $v$ for $i=1$ is understood to be $w(k+1)$. Therefore

$$
\left(V(p), W_{i, j}(p)\right) \equiv \begin{cases}(\{w(k+1)\},\{w(j)\}) & \text { for } p=i \\ (\{w(k+1), w(i)\},\{w(j), w(p)\}) & \text { for } i<p<j\end{cases}
$$

Here $w(k+1) \leq w(j)<w(i)$ by (8.3) because $j \leq k+1$. Therefore, it follows from Lemma 8.1 that (8.2) is equivalent to $w(i)<w(p)$ for $i<p<j$, proving (1).

(2) In this case we have

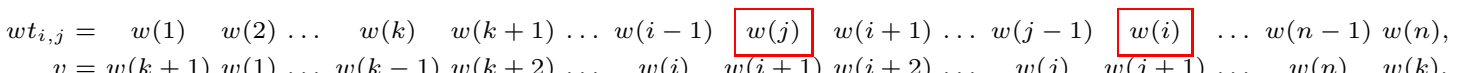

Therefore

$$
\left(V(p), W_{i, j}(p)\right) \equiv(\{w(p+1), w(i)\},\{w(j), w(k)\}) \quad \text { for } i \leq p<j .
$$

Here $w(j)<w(i) \leq w(k)$ by (8.3) because $k \leq i$. Therefore, it follows from Lemma 8.1 that (8.2) is equivalent to $w(p+1) \leq w(j)$ for $i \leq p<j$, proving $(2)$.

(3) In this case we have

$$
\begin{aligned}
& w t_{i, j}=w(1) \quad w(2) \ldots w(i-1) \quad w(j) \quad w(i+1) \ldots \quad w(k) \quad w(k+1) \ldots w(j-1) \quad w(i) \quad \ldots w(n-1) w(n), \\
& v=w(k+1) w(1) \ldots w(i-2) w(i-1) \quad w(i) \quad \ldots w(k-1) \quad w(k+2) \ldots w(j-2) w(j-1) \ldots \quad w(n) \quad w(k) .
\end{aligned}
$$

Therefore

$$
\left(V(p), W_{i, j}(p)\right) \equiv \begin{cases}(\{w(k+1)\},\{w(j)\}) & \text { for } p=i, \\ (\{w(k+1), w(i)\},\{w(j), w(p)\}) & \text { for } i<p \leq k, \\ (\{w(p+1), w(i)\},\{w(j), w(k)\}) & \text { for } k<p<j .\end{cases}
$$

First we treat the case for $i \leq p \leq k$. Note that $w(k+1)<w(i)$ by (8.3) because $i \leq k$. Therefore, it follows from Lemma 8.1 that (8.2) is equivalent to

$$
w(k+1) \leq w(j) \text { and } w(i) \leq w(p) \text { for } i \leq p \leq k .
$$

As for the case when $k<p<j$, note that $w(j)<w(k)$ by (8.3) because $k+1<j$. Therefore, it follows from Lemma 8.1 that (8.2) is equivalent to

$$
w(p+1) \leq w(j) \text { for } k<p<j \text { and } w(i) \leq w(k) .
$$

Inequalities (8.4) and (8.5) prove case (3).

In case (1), the latter inequalities in (8.3) ensure the existence of the desired $j$ for each $i$ and such $j$ is unique for each $i$; so there are exactly $k$ desired pairs $(i, j)$ in case (1). The same is true for case (2) with the role of $i$ and $j$ interchanged. Namely, for each $j$ there exists a unique desired $i$ where the existence of $i$ for the $j$ is ensured by the former inequalities in (8.3); so there are exactly $n-k$ desired pairs $(i, j)$ in case (2). However, cases (1) and (2) have one overlap, that is, the case $(i, j)=(k, k+1)$ and this case satisfies the required condition. Therefore, we obtain exactly $n-1$ coatoms of $[v, w]$ from cases (1) and (2). This proves the last statement in the proposition. 
Remark 8.5. The number of the pairs $(i, j)$ in case (3) is at most $(k-1)(n-k-1)$ and the following example attains the maximum:

$$
\begin{aligned}
& w=[n-k+1, n-k+2, \ldots, \quad n, 1,2, \ldots, n-k-1, n-k], \\
& v=[\quad 1, n-k+1, \ldots, n-1,2,3, \ldots, \quad n-k, \quad n] .
\end{aligned}
$$

Therefore, the Bruhat interval $[v, w]$, which is of length $n-1$, has

$$
(n-1)+(k-1)(n-k-1)=k(n-k)
$$

many coatoms. Note that $k(n-k) \leq\left\lfloor n^{2} / 4\right\rfloor$, and the equality is attained when $k=\lfloor n / 2\rfloor$. It is shown in [14, Theorem in $\S 1$ ] that the number of coatoms of any Bruhat interval of length $n-1$ is at most $\left\lfloor n^{2} / 4\right\rfloor$ and that the maximum can be attained by the above example.

Two types of $\left(V(p), W_{i, j}(p)\right)$ appear for case (3) in the above proof and each appears for cases (1) and (2) respectively. This implies that it suffices to treat case (3) essentially.

Case II. In this case we have

$$
\begin{aligned}
w & =v\left(s_{k} \cdots s_{n-1}\right)\left(s_{k-1} \cdots s_{1}\right), \quad \text { equivalently } \\
v & =w\left(s_{1} \cdots s_{k-1}\right)\left(s_{n-1} \cdots s_{k}\right) .
\end{aligned}
$$

Since $\ell(w)-\ell(v)=n-1$, we have

$$
\begin{aligned}
& w(2), w(3), \ldots, w(k)<w(1), \\
& w(n)<w(1), w(k+1), \ldots, w(n-1) .
\end{aligned}
$$

Proposition 8.6. In Case II, $w t_{i, j}$ is a coatom of $[v, w]$ if and only if one of the following is satisfied:

(1) If $1 \leq i<j \leq k$, then $w(p)<w(j)$ for every $i<p<j$.

(2) If $k<i<j \leq n$, then $w(i)<w(p)$ for every $i<p<j$.

(3) If $1 \leq i \leq k<j \leq n$, then $w(p)<w(j)$ for every $i<p \leq k$ and $w(i)<w(p)$ for every $k<p<j$.

Moreover, $Q_{v, w}$ is a cube in Case II if and only if there is only one pair $(i, j)$ in (3).

Proof. Suppose that $1 \leq i<k$ and $k+1<j \leq n$. Then we have

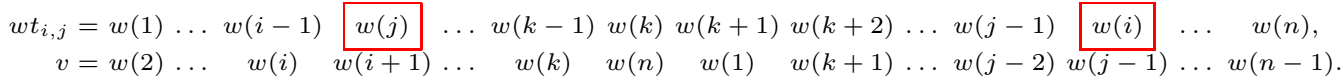

Therefore

$$
\left(V(p), W_{i, j}(p)\right) \equiv \begin{cases}(\{w(p+1), w(i)\},\{w(j), w(1)\}) & \text { for } i \leq p<k \\ (\{w(n), w(i)\},\{w(j), w(1)\}) & \text { for } p=k \\ (\{w(n), w(i)\},\{w(j), w(p)\}) & \text { for } k<p<j .\end{cases}
$$

Here $w(n) \leq w(j)<w(i) \leq w(1)$ by (8.6) because $1 \leq i<k$ and $k+1<j \leq n$. Therefore, it follows from Lemma 8.1 that $(8.2)$ is equivalent to

$$
\begin{aligned}
w(p+1)<w(j) & \text { for } i \leq p<k, \\
w(i)<w(p) & \text { for } k<p<j,
\end{aligned}
$$

proving the assertion when $1 \leq i<k$ and $k+1<j \leq n$.

One can see that the same argument works for the remaining cases with a little modification. For instance, when $1 \leq i<j \leq k$ (i.e., case (1) in the proposition), only the first type in (8.7) occurs and when $k<i<j \leq n$ (i.e., case (2) in the proposition), only the third type in (8.7) occurs. The cases where $i=k$ or $j=k+1$ in case (3) remain. When $i=k$ and $j=k+1$, only the second type 
in (8.7) appears and when $i=k$ and $k+1<j \leq n$, the second and third types in (8.7) appear and when $1 \leq i<k$ and $j=k+1$, the first and second types in (8.7) appear.

In case (1), the former inequalities in (8.6) ensure the existence of the desired $i$ for each $j$ and such $i$ is unique for the $j$; so there are exactly $k-1$ pairs $(i, j)$ in case (1). The same is true for case (2) with the role of $i$ and $j$ interchanged. Namely, for each $i$ there exists a unique desired $j$ where the existence of $j$ for the $i$ is ensured by the latter inequalities in (8.6); so there are exactly $n-k-1$ pairs $(i, j)$ in case (2). Therefore, we obtain exactly $n-2$ coatoms from cases (1) and (2). This proves the last statement in the proposition.

Remark 8.7. If $w(k)>w(k+1)$, then there is only one pair $(i, j)$ in case (3), that is $(i, j)=(k, k+1)$. Therefore, $Q_{v, w}$ is a cube in this case. If $w(k)<w(k+1)$, then it happens that there are more than one pair $(i, j)$ in case (3) but the number of those pairs is at most $\min \{k, n-k\}$ because for each $i$, the desired $j$ is unique if it exists and vice versa. The following examples attain the maximum $\min \{k, n-k\}$ :

when $k>n-k$,

$w=[n, n-2, \ldots, n-2(n-k), 2 k-n-1,2 k-n-2, \ldots, 1, n-1, n-3, \ldots, n-1-2(n-k-1)]$

where $w(k)=1$, and when $k \leq n-k$,

$$
w=[n, n-2, \ldots, n-2(k-1), n-1, n-3, \ldots, n-1-2(k-1), n-2 k, n-2 k-1, \ldots, 1]
$$

where $w(n)=1$. For instance

$$
\begin{array}{ll}
w=[10,8,6,4,3,2,1,9,7,5] & \text { when }(n, k)=(10,7), \\
w=[10,8,6,4,2,9,7,5,3,1] & \text { when }(n, k)=(10,5), \\
w=[10,8,6,9,7,5,4,3,2,1] & \text { when }(n, k)=(10,3) .
\end{array}
$$

Case III. In this case we have

$$
\begin{aligned}
w & =v\left(s_{k} \cdots s_{n-1}\right)\left(s_{1} \cdots s_{k-1}\right), \quad \text { equivalently } \\
v & =w\left(s_{k-1} \cdots s_{1}\right)\left(s_{n-1} \cdots s_{k}\right) .
\end{aligned}
$$

Since $\ell(w)-\ell(v)=n-1$, we have

$$
\begin{aligned}
& w(k)<w(1), \ldots, w(k-1), \\
& w(n)<w(k-1), w(k+1), \ldots, w(n-1) .
\end{aligned}
$$

We note that $w(k)=1$ or $w(n)=1$. Indeed, if $w(k)<w(n)$ (respectively, $w(n)<w(k)$ ), then it follows from (8.8) that $w(k)=1$ (respectively, $w(n)=1$ ).

Proposition 8.8. In Case III, $w t_{i, j}$ is a coatom of $[v, w]$ if and only if one of the following is satisfied:

(1) If $1 \leq i<j \leq k$ or $k<i<j \leq n$, then $w(i)<w(p)$ for every $i<p<j$,

(2) If $i=k<j \leq n$, then $w(i)<w(p)$ for every $i<p<j$ and $w(n)=1$,

(3) If $1 \leq i<k<j \leq n$, then $w(i)<w(p)$ for every $i<p<j$ with $p \neq k$ and $w(k)<w(j)$.

Moreover, $Q_{v, w}$ is a cube in Case III if and only if there is no (respectively, only one) pair $(i, j)$ in (3) when $w(n)=1$ (respectively, $w(k)=1)$.

Proof. Suppose that $1 \leq i<k$ and $k+1<j \leq n$. Then we have

$$
\begin{aligned}
& w t_{i, j}=w(1) w(2) \ldots w(i-1) \quad w(j) \quad w(i+1) \ldots w(k-1) w(k) w(k+1) w(k+2) \ldots w(j-1) \quad w(i) \quad \ldots \quad w(n) \\
& v=w(k) w(1) \ldots w(i-2) w(i-1) \quad w(i) \quad \ldots w(k-2) w(n) w(k-1) w(k+1) \ldots w(j-2) w(j-1) \ldots w(n-1) .
\end{aligned}
$$


Therefore

$$
\left(V(p), W_{i, j}(p)\right) \equiv \begin{cases}(\{w(k)\},\{w(j)\}) & \text { for } p=i, \\ (\{w(k), w(i)\},\{w(j), w(p)\}) & \text { for } i<p<k, \\ (\{w(n), w(i)\},\{w(j), w(k-1)\}) & \text { for } p=k, \\ (\{w(n), w(i)\},\{w(j), w(p)\}) & \text { for } k<p<j .\end{cases}
$$

Here $w(k)<w(i)$ and $w(n) \leq w(j)<w(i)$ by (8.8) because $i<k$ and $k+1<j \leq n$. Therefore, it follows from Lemma 8.1 that 8.2 is equivalent to

$$
\begin{aligned}
& w(k)<w(j), \\
& w(i)<w(p) \quad \text { for } i<p<k \text { or } k<p<j,
\end{aligned}
$$

proving the assertion when $1 \leq i<k$ and $k+1<j \leq n$.

Similarly to the proof of Proposition 8.6 one can see that the same argument works for the remaining cases with a little modification.

For each $1 \leq i<k$ (respectively, $k<i<n$ ), the desired $j$ in case (1) is unique and the existence of such $j$ is ensured by the former (respectively, latter) inequalities in (8.8); so we obtain $n-2$ coatoms from case (1). Since the desired $j$ in case (2) is also unique, we obtain one coatom from case (2) when $w(n)=1$ and none when $w(k)=1$. Therefore, we obtain $n-1$ (respectively, $n-2)$ coatoms from cases (1) and (2) when $w(n)=1$ (respectively, $w(k)=1$ ). This proves the last statement in the proposition.

Remark 8.9. The number of coatoms in case (3) is at most $k$ (respectively, $k-1$ ) when $w(n)=1$ (respectively, $w(k)=1$ ) and the following examples attain the maximum:

$$
\begin{array}{ll}
w=[n-k+2, n-k+3, \ldots, n, 2,3,4, \ldots, n-k+1,1] & \text { when } w(n)=1, \\
w=[n-k+2, n-k+3, \ldots, n, 1,3,4, \ldots, n-k+1,2] & \text { when } w(k)=1 .
\end{array}
$$

\section{REFERENCES}

[1] A. V. Aho, M. R. Garey, and J. D. Ullman. The transitive reduction of a directed graph. SIAM J. Comput., 1(2):131-137, 1972.

[2] G. Araujo-Pardo, M. Del Río-Francos, M. López-Dudet, D. Oliveros, and E. Schulte. The graphicahedron. European J. Combin., 31(7):1868-1879, 2010.

[3] M. Audin. Torus actions on symplectic manifolds, volume 93 of Progress in Mathematics. Birkhäuser Verlag, Basel, revised edition, 2004.

[4] A. Björner and F. Brenti. Combinatorics of Coxeter groups, volume 231 of Graduate Texts in Mathematics. Springer, New York, 2005.

[5] M. Brion, Lectures on the geometry of flag varieties, Topics in cohomological studies of algebraic varieties, Trends Math., 33-85, Birkhäuser, Basel, 2005.

[6] D. A. Cox, J. B. Little, and H. K. Schenck. Toric varieties, volume 124 of Graduate Studies in Mathematics. American Mathematical Society, Providence, RI, 2011.

[7] C. K. Fan. Schubert varieties and short braidedness. Transform. Groups, 3(1):51-56, 1998.

[8] W. Fulton. Young tableaux, volume 35 of London Mathematical Society Student Texts. Cambridge University Press, Cambridge, 1997. With applications to representation theory and geometry.

[9] M. Grossberg and Y. Karshon. Bott towers, complete integrability, and the extended character of representations. Duke Math. J., 76(1):23-58, 1994.

[10] G. T. Guilbaud and P. Rosenstiehl. Analyse algébrique d'un scrutin. Mathématiques et Sciences humaines, 4:9-33, 1963.

[11] M. Harada, T. Horiguchi, M. Masuda, and S. Park. The volume polynomial of regular semisimple Hessenberg varieties and the Gelfand-Zetlin polytope. Proc. Steklov Inst. Math. to appear.

[12] P. Karuppuchamy. On Schubert varieties. Comm. Algebra, 41(4):1365-1368, 2013.

[13] A. A. Klyachko. Orbits of a maximal torus on a flag space. Funktsional. Anal. i Prilozhen., 19(1):77-78, 1985.

[14] M. Kobayashi. The maximum number of atoms of Bruhat intervals in the symmetric groups. European J. Combin., 32(1):67-71, 2011. 
[15] E. Lee and M. Masuda. Generic torus orbit closures in Schubert varieties. arXiv:1807.02904

[16] M. Masuda and T. E. Panov. Semi-free circle actions, Bott towers, and quasitoric manifolds. Mat. Sb., 199(8):95$122,2008$.

[17] A. Postnikov. Permutohedra, associahedra, and beyond. IMRN, 2009(6):1026-1106, 2009.

[18] C. Procesi. The toric variety associated to Weyl chambers. In Mots, Lang. Raison. Calc., pages 153-161. Hermès, Paris, 1990.

[19] N. P. Reading. On the structure of Bruhat order. ProQuest LLC, Ann Arbor, MI, 2002. Thesis (Ph.D.)-University of Minnesota.

[20] B. E. Tenner. Pattern avoidance and the Bruhat order. J. Combin. Theory Ser. A, 114(5):888-905, 2007.

[21] A. Tsuchiya. personal communications.

[22] E. Tsukerman and L. Williams. Bruhat interval polytopes. Adv. Math., 285:766-810, 2015.

[23] L. Yu and M. Masuda. On descriptions of products of simplices. arXiv:1609.05761.

[24] G. M. Ziegler. Lectures on polytopes, volume 152 of Graduate Texts in Mathematics. Springer-Verlag, New York, 1995.

(E. Lee) Center for Geometry and Physics, Institute for Basic Science (IBS), Pohang 37673, Republic of Korea

E-mail address: eunjeong.lee@ibs.re.kr

(M. Masuda) Department of Mathematics, Graduate School of Science, Osaka City University, SumiyoshiKu, Sugimoto, 558-8585, Osaka, Japan

E-mail address: masuda@osaka-cu.ac.jp

(S. Park) Department of mathematics, Ajou University, Suwon 16499, Republic of Korea

E-mail address: seonjeong1124@gmail.com 